\title{
Selfinjective algebras having a generalized standard family of quasi-tubes maximally saturated by simple and projective modules
}

\author{
Alicja Jaworska-Pastuszak ${ }^{a, *}$, Marta Kwiecień ${ }^{b}$, Andrzej Skowroński ${ }^{a}$ \\ ${ }^{a}$ Faculty of Mathematics and Computer Science, Nicolaus Copernicus University, Chopina 12/18, \\ 87-100 Toruń, Poland \\ ${ }^{b}$ Faculty of Mathematics and Computer Science, University of Warmia and Mazury, ul. Słoneczna \\ 54, 10-710 Olsztyn, Poland
}

\begin{abstract}
We give a complete description of finite dimensional selfinjective algebras over an algebraically closed field whose Auslander-Reiten quiver admits a generalized standard family of quasi-tubes maximally saturated by simple and projective modules. In particular, we show that these algebras are selfinjective algebras of strictly canonical type.
\end{abstract}

MSC: 16D50, 16G20, 16G70

Keywords: selfinjective algebra, canonical algebra, Galois covering, repetitive algebra, Auslander-Reiten quiver, quasi-tube

\section{Introduction and the main results}

Throughout the article $K$ will denote a fixed algebraically closed field. By an algebra is meant an associative finite dimensional $K$-algebra with an identity, which we shall assume (without loss of generality) to be basic and indecomposable. For an algebra $A$, we denote by $\bmod A$ the category of finite dimensional (over $K$ ) right $A$-modules, by ind $A$ its full subcategory formed by the indecomposable modules, and by $\mathrm{D}: \bmod A \rightarrow \bmod A^{\text {op }}$ the standard duality $\operatorname{Hom}_{K}(-, K)$. Given a module $M$ in $\bmod A$, we denote by $[M]$ the image of $M$ in the Grothendieck group $K_{0}(A)$ of $A$. Thus $[M]=[N]$ if and only if the modules $M$ and $N$ have the same simple

\section{*Corresponding author}

E-mail addresses:

jaworska@mat.uni.torun.pl (A. Jaworska-Pastuszak), marta.kwiecien@uwm.edu.pl (M. Kwiecień), skowron@mat.uni.torun.pl (A. Skowroński)

Supported by the Research Grant No. DEC-2011/02/A/ST1/00216 of the National Science Center Poland. 
composition factors including the multiplicities. An algebra $A$ is called selfinjective if $A_{A}$ is an injective module, or equivalently, the projective and injective modules in $\bmod A$ coincide. An important class of selfinjective algebras is formed by the orbit algebras $R / G$, where $R$ is a selfinjective locally bounded $K$-category and $G$ is an admissible group of automorphisms of $R$. Then we have a Galois covering $R \rightarrow R / G$ which frequently allows us to reduce the representation theory of $R / G$ to the representation theory of $R$. In the theory, the selfinjective orbit algebras $\widehat{B} / G$ given by the repetitive categories $\widehat{B}$ of triangular algebras $B$ and infinite cyclic admissible groups $G$ of automorphisms of $\widehat{B}$ are of particular interest. We also note that for the algebras $B$ of finite global dimension, the stable module category $\underline{\bmod } \widehat{B}$ of $\widehat{B}$ is equivalent (as a triangulated category) to the derived category $\mathrm{D}^{b}(\bmod B)$ of bounded complexes over $\bmod B[12]$.

An important combinatorial and homological invariant of the module category $\bmod A$ of an algebra $A$ is its Auslander-Reiten quiver. The Auslander-Reiten quiver $\Gamma_{A}$ describes the structure of the quotient category $\bmod A / \operatorname{rad}_{A}^{\infty}$, where $\operatorname{rad}_{A}^{\infty}$ is the infinite Jacobson radical of $\bmod A$ (the intersection of all powers $\operatorname{rad}_{A}^{i}, i \geqslant 1$, of the Jacobson $\operatorname{radical} \operatorname{rad}_{A}$ of $\bmod A$ ). By a result due to Auslander [5], $A$ is of finite representation type if and only if $\operatorname{rad}_{A}^{\infty}=0$ (see also [15] for an alternative proof of this result). On the other hand, if $A$ is of infinite representation type, then $\left(\operatorname{rad}_{A}^{\infty}\right)^{2} \neq 0$, by a result proved in [8]. In general, it is important to study the behaviour of the components of $\Gamma_{A}$ in the category $\bmod A$. Following [28], a family $\mathcal{C}=\left(\mathcal{C}_{\lambda}\right)_{\lambda \in \Lambda}$ of components of $\Gamma_{A}$ is said to be generalized standard if $\operatorname{rad}_{A}^{\infty}(X, Y)=0$ for all modules $X$ and $Y$ in $\mathcal{C}$. It was proved in [28, (2.3)] that every generalized standard family $\mathcal{C}$ of components in $\Gamma_{A}$ is almost periodic, that is, all but finitely many DTr-orbits in $\mathcal{C}$ are periodic. In particular, for a selfinjective algebra $A$, every infinite generalized standard component $\mathcal{C}$ of $\Gamma_{A}$ is either acyclic with finitely many DTr-orbits or a quasi-tube (the stable part $\mathcal{C}^{s}$ of $\mathcal{C}$ is a stable tube $\mathbb{Z} \mathbb{A}_{\infty} /\left(\tau^{r}\right)$, for some $r \geqslant 1$ ).

In the paper we are concerned with the structure of selfinjective algebras $A$ for which the Auslander-Reiten quiver $\Gamma_{A}$ admits a generalized standard component. A distinguished class of such algebras is formed by the selfinjective algebras of finite representation type. By general theory (see [30, Section 3]) these algebras are socle deformations of the orbit algebras $\widehat{B} / G$, for tilted algebras $B$ of Dynkin type and infinite cyclic groups $G$ of automorphisms of $\widehat{B}$. Further, it was proved in [32, 33, 34] that every selfinjective algebra $A$ having an acyclic generalized standard component in $\Gamma_{A}$ is of the form $\widehat{B} / G$, for a tilted algebra $B$ of Euclidean or wild type and an infinite cyclic group $G$ of automorphisms of $\widehat{B}$. On the other hand, the description of selfinjective algebras $A$ whose Auslander-Reiten quiver admits a generalized standard quasi-tube is an exciting but difficult problem. Namely, every algebra $\Lambda$ 
is a quotient algebra of a selfinjective algebra $A$ with $\Gamma_{A}$ having a generalized standard stable tube (see [28], [29]). We refer to [6], [13], [14], [16] for some work on the structure of selfinjective algebras having generalized standard families of quasi-tubes.

In order to formulate the main result of the paper we need to present some concept.

Let $A$ be an algebra. Recall that a smooth quasi-tube is a quasi-tube whose all nonstable vertices are projective-injective. For a smooth quasi-tube $\mathcal{T}$ of $\Gamma_{A}$ we denote by $s(\mathcal{T})$ the number of simple modules in $\mathcal{T}$, by $p(\mathcal{T})$ the number of projective modules in $\mathcal{T}$, and by $r(\mathcal{T})$ the rank of the stable tube $\mathcal{T}^{s}$. Obviously, if $A$ is selfinjective then each quasi-tube is smooth. Moreover, in this case we know that $s(\mathcal{T})+p(\mathcal{T}) \leqslant r(\mathcal{T})-1$ for any quasi-tube $\mathcal{T}$ in $\Gamma_{A}\left[23\right.$. A family $\mathcal{C}=\left(\mathcal{C}_{\lambda}\right)_{\lambda \in \Lambda}$ of smooth quasi-tubes in $\Gamma_{A}$ is said to be maximally saturated by simple and projective modules if there exist two simple right $A$-modules $S$ and $T$ which are not in $\mathcal{C}$ and the following conditions are satisfied:

$(\mathrm{MS} 1) s\left(\mathcal{C}_{\lambda}\right)+p\left(\mathcal{C}_{\lambda}\right)=r\left(\mathcal{C}_{\lambda}\right)-1$ for any $\lambda \in \Lambda$;

(MS2) the simple composition factors of indecomposable modules in $\mathcal{C}$ are $S, T$, the simple modules in $\mathcal{C}$, and the socles and tops of indecomposable projective modules in $\mathcal{C}$;

(MS3) $\mathcal{C}=\left(\mathcal{C}_{\lambda}\right)_{\lambda \in \Lambda}$ consists of all quasi-tubes such that $\mathcal{C}_{\lambda}$ admits an indecomposable module $E_{\lambda}$ with $\operatorname{soc} E_{\lambda}=S$ and top $E_{\lambda}=T$.

In particular, if $p\left(\mathcal{C}_{\lambda}\right)=0$ for any $\lambda \in \Lambda$, we say that a family $\mathcal{C}=\left(\mathcal{C}_{\lambda}\right)_{\lambda \in \Lambda}$ of stable tubes is maximally saturated by simple modules.

The following main result of the paper describes the structure of all selfinjective algebras whose Auslander-Reiten quiver admits a generalized standard family of quasi-tubes maximally saturated by simple and projective modules.

THEOREM 1.1. Let $A$ be a basic, connected, finite dimensional selfinjective algebra over an algebraically closed field $K$. The following statements are equivalent.

(i) $\Gamma_{A}$ admits a generalized standard family $\mathcal{C}=\left(\mathcal{C}_{\lambda}\right)_{\lambda \in \Lambda}$ of quasi-tubes maximally saturated by simple and projective modules.

(ii) $A$ is isomorphic to an orbit algebra $\widehat{B} / G$ of the repetitive category $\widehat{B}$ of a branch extension $B$ of a canonical algebra $C$ with respect to the canonical $\mathbb{P}_{1}(K)$-family of stable tubes of $\Gamma_{C}$ and $G$ is an infinite cyclic group of automorphisms of $\widehat{B}$ of one of the forms:

(a) $G=\left(\varphi \nu_{\widehat{B}}\right)$, for $\varphi$ a strictly positive automorphism of $\widehat{B}$, 
(b) $G=\left(\varphi \nu_{\widehat{B}}\right)$, for $B$ a canonical algebra and $\varphi$ a rigid automorphism of $\widehat{B}$, where $\nu_{\widehat{B}}$ is the Nakayama automorphism of $\widehat{B}$.

We note that the selfinjective algebras occuring in the second statement of the above theorem form a class of selfinjective algebras of strictly canonical type investigated in [17], [18, [19]. In particular, the structure and homological properties of the Auslander-Reiten quivers of selfinjective algebras of strictly canonical type were described in [17].

The following direct consequence of Theorem 1.1 and [24, Theorem 2] provides a characterization of the trivial extensions of the canonical algebras by their minimal injective cogenerators.

Corollary 1.2. Let $A$ be a basic, connected, finite dimensional symmetric algebra over an algebraically closed field $K$. The following statements are equivalent.

(i) $\Gamma_{A}$ admits a generalized standard family $\mathcal{C}=\left(\mathcal{C}_{\lambda}\right)_{\lambda \in \Lambda}$ of quasi-tubes maximally saturated by simple and projective modules.

(ii) $\Gamma_{A}$ admits a generalized standard family $\mathcal{T}=\left(\mathcal{T}_{\lambda}\right)_{\lambda \in \Lambda}$ of stable tubes maximally saturated by simple modules.

(iii) $A$ is isomorphic to the trivial extension $B \ltimes D(B)$ of a canonical algebra $B$.

The paper is organized in the following way. In Section 2 we recall the canonical algebras and describe their canonical family of stable tubes. Section 3 presents quasitube enlargements of algebras. In Section 4 we show the needed facts on repetitive algebras and their orbit algebras. Section 5 contains results on selfinjective algebras of strictly canonical type which allow us to state that the implication (ii) $\Rightarrow$ (i) of Theorem 1.1 is true. In Section 6 we complete the proof of Theorem 1.1. The final Section 7 is devoted to some examples illustrating the main results of the paper.

For basic background on the representation theory of algebras applied in the paper we refer to the books [1], 25], [26], 27], 335, [36].

\section{Canonical algebras}

The aim of this section is to introduce the canonical algebras and show that they are exactly the algebras whose Auslander-Reiten quiver contains a faithful generalized standard family of stable tubes maximally saturated by simple modules.

Throughout the paper for a vertex $x$ in the Gabriel quiver $Q_{A}$ of an algebra $A$, by $S(x), P(x)$ and $I(x)$ we denote simple, indecomposable projective and indecomposable injective $A$-module at vertex $x$, respectively. Moreover, by $M=$ 
$\left(M_{a}, M_{\alpha}\right)_{a \in Q_{0}, \alpha \in Q_{1}}$, where $Q_{0}$ is a set of vertices and $Q_{1}$ is a set of arrows in $Q_{A}$, we denote a $K$-linear representation of $Q_{A}$. If $I$ is an admissible ideal of $K Q_{A}$, then by representation $M=\left(M_{a}, M_{\alpha}\right)$ of $Q_{A}$ we will mean a representation satisfying the relations in $I$.

Let $m \geq 2$ be an integer number, $\boldsymbol{p}=\left(p_{1}, \ldots, p_{m}\right)$ a sequence of positive integer numbers and $\boldsymbol{\lambda}=\left(\lambda_{1}, \ldots, \lambda_{m}\right)$ a sequence of pairwise different elements of the projective line $\mathbb{P}_{1}(K)=K \cup\{\infty\}$ normalized in such a way that $\lambda_{1}=\infty$ and $\lambda_{2}=0$. Consider the quiver $\Delta(\boldsymbol{p})$ of the form

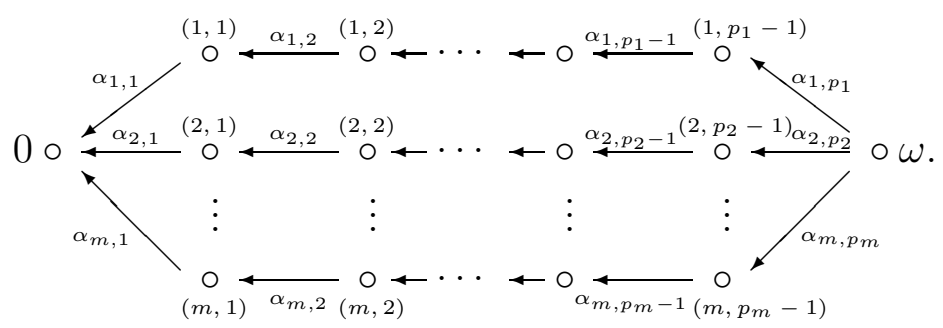

For $m=2, C(\boldsymbol{p}, \boldsymbol{\lambda})$ is defined to be the path algebra $K \Delta(\boldsymbol{p})$ of the quiver $\Delta(\boldsymbol{p})$ over $K$. For $m \geq 3, C(\boldsymbol{p}, \boldsymbol{\lambda})$ is defined to be the quotient algebra $K \Delta(\boldsymbol{p}) / I(\boldsymbol{p}, \boldsymbol{\lambda})$ of the path algebra $K \Delta(\boldsymbol{p})$ by the ideal $I(\boldsymbol{p}, \boldsymbol{\lambda})$ of $K \Delta(\boldsymbol{p})$ generated by the elements

$$
\alpha_{j, p_{j}} \ldots \alpha_{j, 1}+\alpha_{1, p_{1}} \ldots \alpha_{1,1}+\lambda_{j} \alpha_{2, p_{2}} \ldots \alpha_{2,1} \text {, where } j \in\{3, \ldots, m\} .
$$

Following [25], $C(\boldsymbol{p}, \boldsymbol{\lambda})$ is said to be a canonical algebra of type $(\boldsymbol{p}, \boldsymbol{\lambda}), \boldsymbol{p}$ the weight sequence of $C(\boldsymbol{p}, \boldsymbol{\lambda})$, and $\boldsymbol{\lambda}$ the (normalized) parameter sequence of $C(\boldsymbol{p}, \boldsymbol{\lambda})$. It follows from $[25,(3.7)]$ that, for a canonical algebra $C=C(\boldsymbol{p}, \boldsymbol{\lambda})$, the AuslanderReiten quiver $\Gamma_{C}$ of $C$ is of the form

$$
\Gamma_{C}=\mathcal{P}^{C} \cup \mathcal{T}^{C} \cup \mathcal{Q}^{C}
$$

where $\mathcal{P}^{C}$ is a family of components containing all indecomposable projective $C$ modules (hence the unique simple projective $C$-module $S(0)$ associated with the vertex 0 of $\Delta(\boldsymbol{p})), \mathcal{Q}^{C}$ is a family of components containing all indecomposable injective $C$-modules (hence the unique simple injective $C$-module $S(\omega)$ associated with the vertex $\omega$ of $\Delta(\boldsymbol{p}))$, and $\mathcal{T}^{C}=\left(\mathcal{T}_{\lambda}^{C}\right)_{\lambda \in \mathbb{P}_{1}(K)}$ is a canonical $\mathbb{P}_{1}(K)$-family of pairwise orthogonal standard stable tubes separating $\mathcal{P}^{C}$ from $\mathcal{Q}^{C}$ and containing all simple $C$-modules except $S(0)$ and $S(\omega)$. Moreover, if $r_{\lambda}^{C}$ denotes the rank of the stable tube $\mathcal{T}_{\lambda}^{C}$, then $r_{\lambda_{i}}^{C}=p_{i}$, for any $i \in\{1, \ldots, m\}$, and $r_{\lambda}^{C}=1$, for $\lambda \in \mathbb{P}_{1}(K) \backslash\left\{\lambda_{1}, \ldots, \lambda_{m}\right\}$.

Let $C=C(\boldsymbol{p}, \boldsymbol{\lambda})$ be a canonical algebra. We recall the description of modules lying on the mouths of stable tubes of the canonical $\mathbb{P}_{1}(K)$-family $\mathcal{T}^{C}=\left(\mathcal{T}_{\lambda}^{C}\right)_{\lambda \in \mathbb{P}_{1}(K)}$ of $\Gamma_{C}$ : 
(a) For $\lambda=\lambda_{1}=\infty$, the mouth of $\mathcal{T}_{\lambda}^{C}=\mathcal{T}_{\infty}^{C}$ consists of the simple $C$-modules $S(1,1), \ldots, S\left(1, p_{1}-1\right)$ at the vertices $(1,1), \ldots,\left(1, p_{1}-1\right)$ of $\Delta(\boldsymbol{p})$, if $p_{1} \geq 2$, and the nonsimple $C$-module $E^{(\infty)}$ of the form

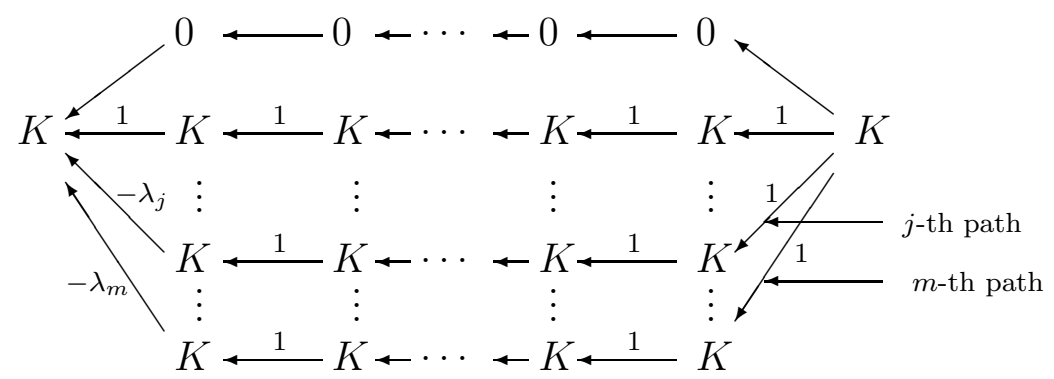

with $j \in\{3, \ldots, m\}$;

(b) For $\lambda=\lambda_{2}=0$, the mouth of $\mathcal{T}_{\lambda}^{C}=\mathcal{T}_{0}^{C}$ consists of the simple $C$-modules $S(2,1), \ldots, S\left(2, p_{2}-1\right)$ at the vertices $(2,1), \ldots,\left(2, p_{2}-1\right)$ of $\Delta(\boldsymbol{p})$, if $p_{2} \geq 2$, and the nonsimple $C$-module $E^{(0)}$ of the form

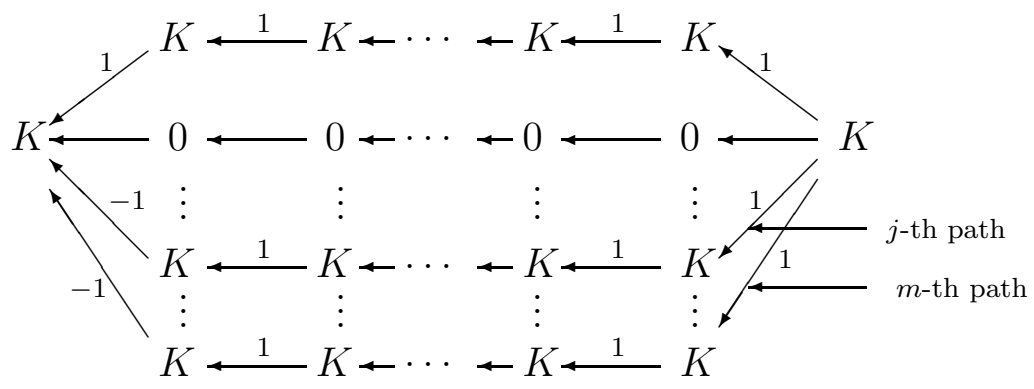

with $j \in\{3, \ldots, m\}$;

(c) For $\lambda=\lambda_{j}$ with $j \in\{3, \ldots, m\}$, the mouth of $\mathcal{T}_{\lambda}^{C}$ consists of the simple $C$ modules $S(j, 1), \ldots, S\left(j, p_{j}-1\right)$ at the vertices $(j, 1), \ldots,\left(j, p_{j}-1\right)$ of $\Delta(\boldsymbol{p})$, if $p_{j} \geq 2$, and the nonsimple $C$-module $E^{\left(\lambda_{j}\right)}$ of the form

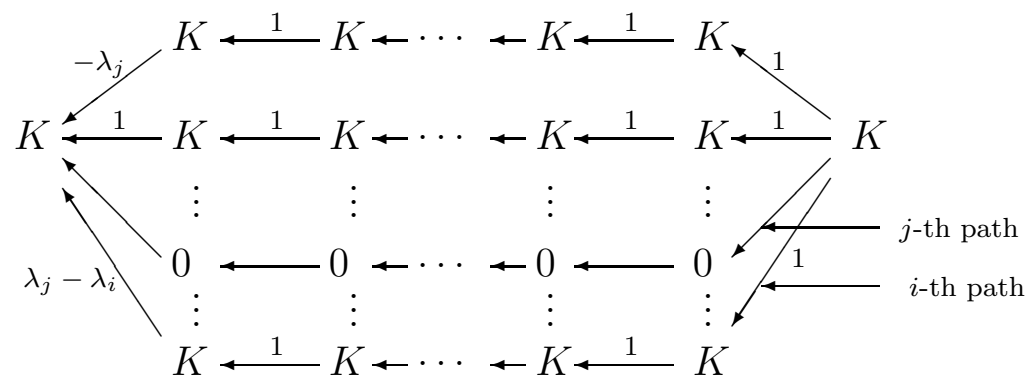

for $i \in\{3, \ldots, m\} \backslash\{j\}$; 
(d) For $\lambda \in \mathbb{P}_{1}(K) \backslash\left\{\lambda_{1}, \ldots, \lambda_{m}\right\}$, the mouth of $\mathcal{T}_{\lambda}^{C}$ consists of one nonsimple $C$-module $E^{(\lambda)}$ of the form

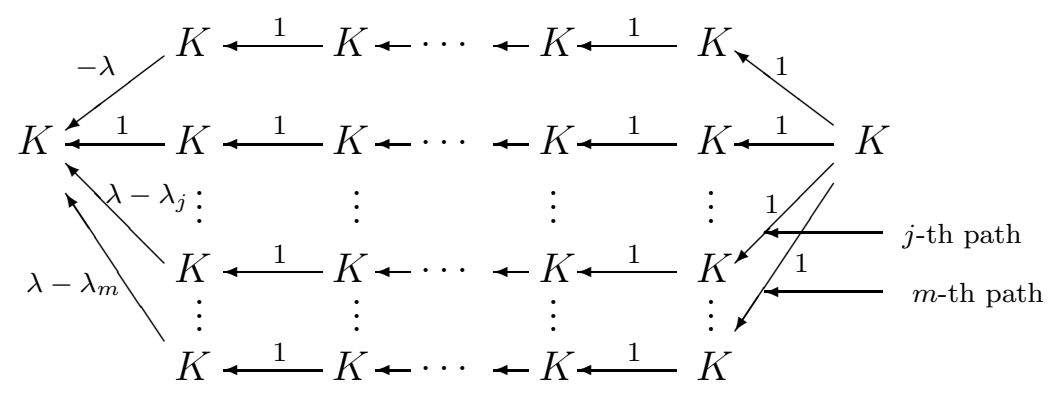

with $j \in\{3, \ldots, m\}$.

The following results describing generalized standard stable tubes of an AuslanderReiten quiver were established in [28, Corollary 5.3].

Proposition 2.1. Let $A$ be an algebra and $\Gamma$ a stable tube of $\Gamma_{A}$. Then the following statements are equivalent.

(i) $\Gamma$ is generalized standard.

(ii) $\Gamma$ is standard.

(iii) The mouth of $\Gamma$ consists of pairwise orthogonal bricks.

(iv) $\operatorname{rad}_{A}^{\infty}(X, X)=0$ for any module $X$ in $\Gamma$.

Recall that an indecomposable $A$-module $X$ is called brick if its endomorphism algebra $\operatorname{End}_{A}(X)$ is isomorphic to $K$.

We now give the characterization of canonical algebras by means of a family of stable tubes which are maximally saturated by simple modules.

THEOREM 2.2. Let $B$ be an algebra. Then the following statements are equivalent.

(i) $\Gamma_{B}$ contains a faithful generalized standard family $\mathcal{C}=\left\{\mathcal{C}_{\lambda}\right\}_{\lambda \in \Lambda}$ of stable tubes maximally saturated by simple modules.

(ii) $B$ is a canonical algebra.

Proof. We proof only that (i) implies (ii), as the other implication is obvious in view of the above description.

Let $n=r k K_{0}(B)$ and $S=S(0), T=S(\omega)$ be simple $B$-modules at the vertices 0 and $\omega$ of the Gabriel quiver $Q_{B}$ of $B$, respectively. Since $\mathcal{C}$ is a generalized standard 
family we have that the modules $S$ and $T$ are not isomorphic and hence $0 \neq \omega$. For a module $M \in \mathcal{C}$, by $l_{\mathcal{C}}(M)$ we shall denote the length of $M$ in the additive category add $\mathcal{C}$ of $\mathcal{C}$ in $\bmod B$, that is, the length $l$ of a chain $M=M_{0} \supset M_{1} \supset \ldots \supset M_{l}=0$ of submodules of $M$ which belong to $\mathcal{C}$ and such that $M_{j-1} / M_{j}$ is a module from the mouth of $\mathcal{C}_{\lambda}$, for $1 \leq j \leq l$ and some $\lambda \in \Lambda$. If $X$ is a module from the mouth of $\mathcal{C}_{\lambda}$, for some $\lambda \in \Lambda$, by $X[j]$ we shall denote a module $M$ which belongs to infinite ray starting at $X$ such that $l_{\mathcal{C}}(M)=j$.

We have two cases to consider.

(1) Assume that the family $\mathcal{C}=\left\{\mathcal{C}_{\lambda}\right\}_{\lambda \in \Lambda}$ consists entirely of homogenous tubes. Since $\mathcal{C}$ is faithful family maximally saturated by simple modules, we have that $S$ and $T$ are the only simple $B$-modules. Moreover, by [28, Lemma 5.9] $\operatorname{pd}_{A} X \leq 1$ and $\operatorname{id}_{A} X \leq 1$ for any indecomposable module $X \in \mathcal{C}$. Therefore, the ordinary quiver $Q_{B}$ of $B$ does not admit any oriented cycle. Then, by (MS3), we conclude that $Q_{B}$ is of the form:

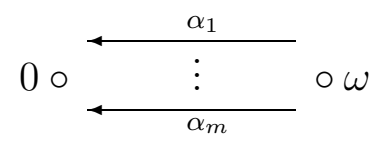

for some $m \geq 1$. Assume that $\alpha_{1}, \ldots, \alpha_{m}$ are linearly independent elements of the $K$-vector space $e_{\omega} B e_{0}$ and hence $B$ is the path algebra $K Q_{B}$. Note that $m \geq 2$ since $B$ is representation-infinite. If $m \geq 3$, then $B$ is wild and $\Gamma_{B}$ does not contain tubes (see [27, XVIII.1.6]). Hence $B$ is the path algebra $K Q_{B}$ of the Kronecker quiver:

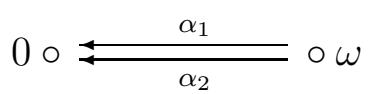

and $\mathcal{C}=\left\{\mathcal{C}_{\lambda}\right\}_{\lambda \in \Lambda}$ is a unique $\mathbb{P}_{1}(K)$-family of homogenous tubes of $\Gamma_{B}$ (see [26, (XI.4.6)]).

(2) Assume that $\left\{\mathcal{C}_{1} \ldots, \mathcal{C}_{m}\right\}$, for some $m \geqslant 1$, is a complete set of stable tubes of rank at least two in the family $\mathcal{C}=\left\{\mathcal{C}_{\lambda}\right\}_{\lambda \in \Lambda}$. Note that all simple modules from $\mathcal{C}$ lie on mouths of these tubes. Since $\mathcal{C}$ is faithful, simple modules from $\mathcal{C}$ together with $S$ and $T$ form the set of all simple $B$-modules. We mention also that the number $m$ of nonhomogenous tubes in $\Gamma_{B}$ is no greater than $n$, by [28, (2.2)].

Let $\mathcal{C}_{i}$, for some $i \in\{1, \ldots, m\}$, be a tube of rank $r\left(\mathcal{C}_{i}\right)=p_{i} \geq 2$. Denote by $S_{(i, 1)}, \ldots, S_{\left(i, p_{i}-1\right)}$ all simple modules which belong to $\mathcal{C}_{i}$ and by $F_{i}$ the remaining module from the mouth of $\mathcal{C}_{i}$. Note that $F_{i}$ satisfies the condition (MS3), otherwise an epimorphism $f: E_{\lambda} \rightarrow T$ shall not factorize by a mouth module (see [26, Lemma $\mathrm{X} .2 .9])$. 
We start with describing the Gabriel quiver $Q_{B}$ of $B$. Since $\mathcal{C}$ is generalized standard family of stable tubes, by Proposition 2.1, we have that

$$
\operatorname{dim}_{K} \operatorname{Ext}_{B}^{1}\left(S^{\prime}, S^{\prime \prime}\right)=\operatorname{dim}_{K} \operatorname{Hom}_{B}\left(S^{\prime \prime}, \tau_{B} S^{\prime}\right)=\left\{\begin{array}{lll}
1, & \text { if } & \tau_{B} S^{\prime}=S^{\prime \prime} \\
0, & \text { if } & \tau_{B} S^{\prime} \neq S^{\prime \prime}
\end{array},\right.
$$

for any simple modules $S^{\prime}, S^{\prime \prime}$ in $\mathcal{C}$. Consider now the tube $\mathcal{C}_{i}$. Without loss of generality we assume that $\tau_{B} S_{(i, l)}=S_{(i, j)}$ if $l=j+1$, for $j \in\left\{1, \ldots, p_{i}-2\right\}$ and $\tau_{B} S_{(i, 1)}=F_{i}, \tau_{B} F_{i}=S_{\left(i, p_{i}-1\right)}$. Observe that:

- $\operatorname{dim}_{K} \operatorname{Ext}_{B}^{1}\left(T, S_{\left(i, p_{i}-1\right)}\right)=\operatorname{dim}_{K} \underline{\operatorname{Hom}}_{B}\left(\tau_{B}^{-} S_{\left(i, p_{i}-1\right)}, T\right)=\operatorname{dim}_{K} \underline{\operatorname{Hom}}_{B}\left(F_{i}, T\right)=$ $=\operatorname{dim}_{K} \operatorname{Hom}_{B}\left(F_{i}, T\right)=1$, since $T=\operatorname{top} F_{i}$,

- $\operatorname{dim}_{K} \operatorname{Ext}_{B}^{1}\left(T, S_{(i, j)}\right)=\operatorname{dim}_{K} \underline{\operatorname{Hom}}_{B}\left(\tau_{B}^{-} S_{(i, j)}, T\right)=\operatorname{dim}_{K} \underline{\operatorname{Hom}}_{B}\left(S_{(i, j+1)}, T\right)=0$, since $S_{(i, j+1)}, T$ are nonisomorphic simple $B$-modules, for $1 \leq j<p_{i}-1$,

- $\operatorname{dim}_{K} \operatorname{Ext}_{B}^{1}\left(S_{(i, 1)}, S\right)=\operatorname{dim}_{K} \overline{\operatorname{Hom}}_{B}\left(S, \tau_{B} S_{(i, 1)}\right)=\operatorname{dim}_{K} \overline{\operatorname{Hom}}_{B}\left(S, F_{i}\right)=1$, because $S=\operatorname{soc} F_{i}$,

- $\operatorname{dim}_{K} \operatorname{Ext}_{B}^{1}\left(S_{(i, j)}, S\right)=\operatorname{dim}_{K} \overline{\operatorname{Hom}}_{B}\left(S, \tau_{B} S_{(i, j)}\right)=\operatorname{dim}_{K} \overline{\operatorname{Hom}}_{B}\left(S, S_{(i, j-1)}\right)=0$, since $S, S_{(i, j-1)}$ are nonisomorphic simple $B$-modules, for any $1<j \leq p_{i}-1$,

- $\operatorname{dim}_{K} \operatorname{Ext}_{B}^{1}\left(S_{(i, j)}, T\right)=0$, for any $1 \leq j \leq p_{i}-1$, because $\operatorname{Hom}_{B}\left(T, \tau_{B} S_{(i, j)}\right)=$ $\operatorname{Hom}_{B}\left(T, S_{(i, j-1)}\right)=0$, for $j>1$, and $\operatorname{Hom}_{B}\left(T, \tau_{B} S_{(i, 1)}\right)=\operatorname{Hom}_{B}\left(T, F_{i}\right)=$ $\operatorname{Hom}_{B}\left(\operatorname{top} F_{i}, F_{i}\right)=0$,

- $\operatorname{dim}_{K} \operatorname{Ext}_{B}^{1}\left(S, S_{(i, j)}\right)=0$, for any $1 \leq j \leq p_{i}-1$, because $\operatorname{Hom}_{B}\left(\tau_{B}^{-} S_{(i, j)}, S\right)=$ $\operatorname{Hom}_{B}\left(S_{(i, j+1)}, S\right)=0$, for $j<p_{i}-1$, and $\operatorname{Hom}_{B}\left(\tau_{B}^{-} S_{\left(i, p_{i}-1\right)}, S\right)=\operatorname{Hom}_{B}\left(F_{i}, S\right)=$ $\operatorname{Hom}_{B}\left(F_{i}, \operatorname{soc}_{i}\right)=0$.

Denote by $(i, j)$, for $1 \leq i \leq m$ and $1 \leq j \leq p_{i}-1$, the vertex of $Q_{B}$ for which $S_{(i, j)}$ is the simple $B$-module at this vertex, that is $S_{(i, j)}=S(i, j)$. Then by [1, (III.2.12)], we obtain that $Q_{B}$ has a subquiver $Q$ of the form

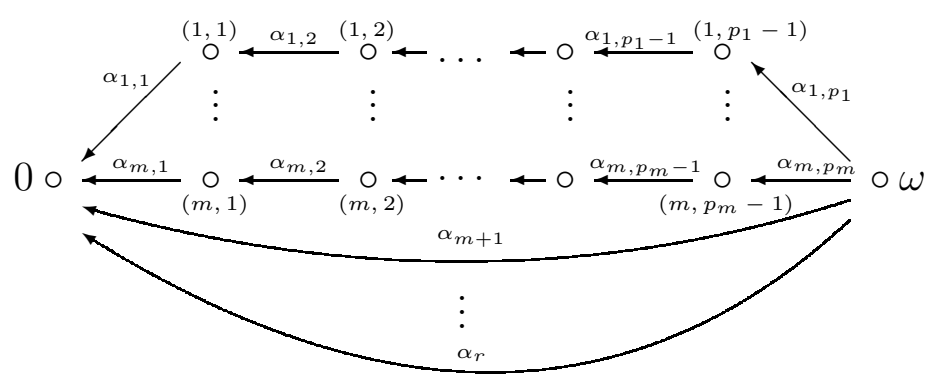

where $r \geq m$, there are no other vertices in $Q_{B}$, no other arrows starting at or ending in vertices $(i, j)$ and no other arrows starting at $\omega$ and ending in 0 . Since $\mathcal{C}$ 
form a hereditary family of modules in $\bmod B$ (see [28, Lemma 5.9]), then any path $\alpha_{i, p_{i}} \ldots \alpha_{i, 1}$, for $1 \leq i \leq m$, is a nonzero element of $B$, and $Q$ does not admit any oriented cycle. Therefore, we conclude that $Q_{B}=Q$.

Consider now the algebra $B^{\prime}=e B e$, where $e=e_{0}+e_{\omega}$. There is the canonical restriction functor

$$
\operatorname{res}_{e}: \bmod B \rightarrow \bmod B^{\prime}
$$

which assigns to a module $M$ in $\bmod B$ the $\operatorname{module}_{e}(M)=M e$ in $\bmod B^{\prime}$ and to a homomorphism $f: M \rightarrow N$ in $\bmod B$ its restriction $\operatorname{res}_{e}(f): \operatorname{res}_{e}(M) \rightarrow \operatorname{res}_{e}(N)$ to $M e$. Note that $Q_{B^{\prime}}$ is an enlarged Kronecker quiver with $r$ arrows

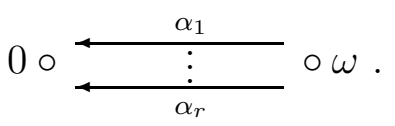

Now we apply the functor $\operatorname{res}_{e}$ to the additive subcategory $\operatorname{add}\left(\mathcal{C}_{i}\right)$ of $\bmod B$. Observe that $\operatorname{res}_{e}\left(S_{(i, 1)}\right)=\operatorname{res}_{e}\left(S_{(i, 2)}\right)=\ldots=\operatorname{res}_{e}\left(S_{\left(i, p_{i}-1\right)}\right)=0$. Moreover, since $F_{i}=\tau_{B} S_{(i, 1)}$, we have $\operatorname{res}_{e}\left(F_{i}\right)=Z=\left(Z_{x}, Z_{\alpha}\right)_{x, \alpha}$, where $Z_{x}=K$ for the vertices $0, \omega$, and $Z_{\alpha}=0$ only for $\alpha=\alpha_{i}$. Clearly, $\operatorname{res}_{e}\left(F_{i}\right)$ is an indecomposable $B^{\prime}$-module. We shall now fix $E_{i}=S_{(i, 1)}\left[p_{i}\right]$. Let $M_{i}=F_{i}\left[p_{i}+1\right]$. Since $\tau_{B}^{-} F_{i}=S_{(i, 1)}$, there exists an exact sequence of $B$-modules

$$
0 \longrightarrow F_{i} \stackrel{f}{\longrightarrow} M_{i} \stackrel{g}{\longrightarrow} E_{i} \longrightarrow 0 \text {. }
$$

Then we have an exact sequence of $B^{\prime}$-modules

$$
0 \longrightarrow \operatorname{res}_{e}\left(F_{i}\right) \stackrel{\operatorname{res}_{e}(f)}{\longrightarrow} \operatorname{res}_{e}\left(M_{i}\right) \stackrel{\operatorname{res}_{e}(g)}{\longrightarrow} \operatorname{res}_{e}\left(E_{i}\right) \longrightarrow 0
$$

because the restriction functor res $_{e}$ is exact (see [1, Theorem I.6.8]). Observe that $\operatorname{res}_{e}\left(E_{i}\right)=\operatorname{res}_{e}\left(F_{i}\right)$. Moreover, $W=\operatorname{res}_{e}\left(M_{i}\right)$ is indecomposable, since $M_{i}$ is indecomposable and, for $j \in\{1, \ldots, m\}, W_{\alpha_{j}}: K^{2} \rightarrow K^{2}$ is given by $W_{\alpha_{j}}=\left(M_{i}\right)_{\varrho}$, where $\varrho=\alpha_{j, p_{j}} \alpha_{j, p_{j-1}} \ldots \alpha_{j, 2} \alpha_{j, 1}$.

We are now in the position to show that $\operatorname{res}_{e}(f)$ is a left almost split homomorphism in $\bmod B^{\prime}$. Since $\operatorname{res}_{e}\left(M_{i}\right)$ is indecomposable and $\operatorname{res}_{e}(g) \neq 0, \operatorname{res}_{e}(f)$ is not a section. Let $u: \operatorname{res}_{e}\left(F_{i}\right) \rightarrow U$, for some indecomposable $B^{\prime}$-module $U$, be a non-zero homomorphism which is not a section. Since $\operatorname{End}_{B^{\prime}}\left(\operatorname{res}_{e}\left(F_{i}\right)\right) \cong K$, it follows that $U \nRightarrow \operatorname{res}_{e}\left(F_{i}\right)$. Invoking the extension functor $L_{e}: \bmod B^{\prime} \rightarrow \bmod B$, $L_{e}(-)=\operatorname{Hom}_{B^{\prime}}(B e,-)$, which is right adjoint to $\operatorname{res}_{e}$, we obtain that there exists a homomorphism $v: F_{i} \rightarrow L_{e}(U)$ of $B$-modules such that $u=\operatorname{res}_{e}(v)$. The functor $L_{e}$ preserves indecomposability of modules, thus $L_{e}(U)$ is indecomposable. Moreover, there is a functorial isomorphism $\operatorname{res}_{e} L_{e} \cong 1_{\bmod B}$ (see [1, Theorem I.6.8]). Hence, $v$ is not a section. We claim that $L_{e}(U)$ is not of the form $F_{i}[j]$ for $j \in\left\{1, \ldots, p_{i}\right\}$. Observe that $\operatorname{res}_{e}\left(F_{i}[j]\right)=\operatorname{res}_{e}\left(F_{i}\right)$ for any $j \in\left\{1, \ldots, p_{i}\right\}$. Then the claim follows 
from the facts that $\operatorname{res}_{e} L_{e}(U) \cong U$ and $U \nRightarrow \operatorname{res}_{e}\left(F_{i}\right)$. Therefore, $v: F_{i} \rightarrow L_{e}(U)$ is a composition $w f_{p_{i}} \ldots f_{1}$ for some homomorphism $w: M_{i} \rightarrow L_{e}(U)$ and irreducible homomorphisms $f_{j}: F_{i}[j] \rightarrow F_{i}[j+1]$ for $j \in\left\{1, \ldots, p_{i}\right\}$ (see [1, Lemma IV.5.1]). Thus $v$ factorizes through $M_{i}=F_{i}\left[p_{i}+1\right]$. Invoking now the restriction functor res $e_{e}$, we conclude that $u=\operatorname{res}_{e}(v)$ factorizes through $\operatorname{res}_{e}\left(M_{i}\right)$. It shows that $u$ is left almost split and, by [1, Theorem IV.1.13],

$$
0 \longrightarrow \operatorname{res}_{e}\left(F_{i}\right) \stackrel{\operatorname{res}_{e}(f)}{\longrightarrow} \operatorname{res}_{e}\left(M_{i}\right) \stackrel{\operatorname{res}_{e}(g)}{\longrightarrow} \operatorname{res}_{e}\left(E_{i}\right) \longrightarrow 0
$$

is an almost split sequence in $\bmod B^{\prime}$. Consequently, the image of $\mathcal{C}_{i}$ by the functor res $_{e}$ is a homogenous tube of $\Gamma_{B^{\prime}}$. Again, by [27, (XVIII.1.6)], we conclude that $r=2$ since $B^{\prime}$ is a tame algebra. Hence $\operatorname{dim}_{K} e_{\omega} B e_{0}=2$.

Let us denote by $\varrho_{i}$ the path $\alpha_{i, p_{i}} \ldots \alpha_{i, 1}$ if $1 \leq i \leq m$, and the arrow $\alpha_{i}$ if $m+1 \leq i \leq r$. Assume now that $\lambda \varrho_{i}=\varrho_{j}$ for some $j \neq i, 1 \leq j, i \leq r$ and non-zero $\lambda \in K$. Fix a stable tube $\mathcal{C}_{i} \in \mathcal{C}$ which contains the simple modules $S(x)$ at the vertices $x \in\left\{(i, 1),(i, 2), \ldots,\left(i, p_{i}-1\right)\right\}$. For simplicity, denote the unique non-simple module $F_{i}$ which lies on the mouth of $\mathcal{C}_{i}$ by $F$. Observe that $F_{\alpha_{i, 1}}=\ldots=F_{\alpha_{i, p_{i}}}=0$ and hence $F_{\varrho_{i}}=F_{\alpha_{i, p_{i}} \ldots \alpha_{i, 1}}=0$. Then $F_{\varrho_{j}}=F_{\varrho_{\varrho_{i}}}=0$ which is impossible since $F=\tau_{B} S_{(i, 1)}$.

Without loss of generality we may now assume that $\varrho_{1}, \varrho_{2}$ form a basis of $e_{\omega} B e_{0}$. From the above consideration we conclude that the equations which describe $\varrho_{3}, \ldots, \varrho_{m}, \alpha_{m+1}, \ldots, \alpha_{r}$ define the set $\Omega$ of generic relations (in the sense of [25]) in $K Q_{B}$. Then, by [25, (3.7)], $B \cong K Q_{B} /\langle\Omega\rangle$ is a canonical algebra and $\mathcal{C}$ is a canonical separating $\mathbb{P}_{1}(K)$-family of stable tubes of $\Gamma_{B}$. Note that $B$ is a canonical algebra of type $(\boldsymbol{p}, \boldsymbol{\lambda})$, where the weight sequence $\boldsymbol{p}$ contains a subsequence $\left(p_{1}, \ldots, p_{m}\right)$ and the parameter sequence $\boldsymbol{\lambda}$ is determined by relations in $\Omega$. Following [17. Lemma 1.1] (see also [25, (3.7)]), if $m=r \geq 3$, we may assume that $p_{i} \geq 2$ for each $i \in\{1, \ldots, m\}$.

\section{Quasi-tube enlargements of algebras}

In this section we introduce quasi-tube enlargements of algebras which are essential in the paper.

A connected translation quiver $\Gamma$ is said to be a quasi-tube if $\Gamma$ can be obtained from a stable tube by an iterated application of admissible operations (ad1), (ad2), $\left(\operatorname{ad} 1^{*}\right)$, or $\left(\operatorname{ad} 2^{*}\right)$ (see [3, Section 2], 44, Section 2] for details). The following proposition provides a characterization of quasi-tubes in Auslander-Reiten quivers of selfinjective algebras ([20], [21], [37]).

Proposition 3.1. Let $A$ be a selfinjective algebra and $\Gamma$ a connected component of $\Gamma_{A}$. The following statements are equivalent. 
(i) $\Gamma$ is a quasi-tube.

(ii) The stable part $\Gamma^{s}$ of $\Gamma$ is a stable tube.

(iii) $\Gamma$ contains an oriented cycle.

Recall that the stable part $\Gamma^{s}$ of $\Gamma$ is obtained from $\Gamma$ by removing the projectiveinjective modules and the arrows attached to them.

Let $A$ be an algebra and $X$ a module in $\bmod A$. The one-point extension of $A$ by $X$ is the $2 \times 2$-matrix algebra

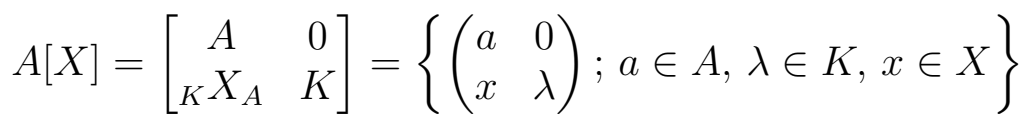

with the usual addition of matrices and the multiplication induced from the canonical $K$-A-bimodule structure ${ }_{K} X_{A}$ of $X$. Dually, the one-point coextension of $A$ by $X$ is the $2 \times 2$-matrix algebra

$$
[X] A=\left[\begin{array}{cc}
K & 0 \\
{ }_{A} D(X)_{K} & A
\end{array}\right]=\left\{\left(\begin{array}{ll}
\lambda & 0 \\
f & a
\end{array}\right) ; a \in A, \lambda \in K, f \in D(X)\right\}
$$

with the usual addition of matrices and the multiplication induced from the canonical $A$-K-bimodule structure ${ }_{A} D(X)_{K}$ of $D(X)=\operatorname{Hom}_{K}\left({ }_{K} X_{A}, K\right)$.

For an algebra $A$ let $\Gamma$ be a generalized standard component of $\Gamma_{A}$. For each indecomposable module $X$ in $\Gamma$ which is a pivot of an admissible operation of type $(\operatorname{ad} 1),(\operatorname{ad} 2),\left(\operatorname{ad} 1^{*}\right)$, or $\left(\operatorname{ad} 2^{*}\right)$, we shall define the corresponding admissible operation on $A$ in such a way that the modified translation quiver $\Gamma^{\prime}$ is a component of the Auslander-Reiten quiver $\Gamma_{A^{\prime}}$ of the modified algebra $A^{\prime}$ (see [3], 4]). Since $\Gamma$ is generalized standard, such a pivot $X$ is necessarily a brick. Suppose that $X$ is the pivot of an admissible operation of type $(\mathrm{ad} 1)$ and that $t$ is a positive integer. Denote $H=H_{t}$ the full $t \times t$ upper triangular matrix algebra over $K$ and by $Y$ the unique indecomposable projective-injective $H$-module, which we consider as a $K$-H-bimodule. Then $A^{\prime}=(A \times H)[X \oplus Y]$ is the required modified algebra. If $X$ is the pivot of an admissible operation of type $(\operatorname{ad} 2)$, then the modified algebra $A^{\prime}$ is defined to be $A^{\prime}=A[X]$. Dually, invoking the one-point coextensions, one defines the modified algebra $A^{\prime}$, if $X$ is a pivot of an admissible operation of type $\left(\operatorname{ad} 1^{*}\right)$ or $\left(\operatorname{ad} 2^{*}\right)$. Then the following fact mentioned above holds (see [3, Section 2]).

Lemma 3.2. The modified translation quiver $\Gamma^{\prime}$ of $\Gamma$ is a component of $\Gamma_{A^{\prime}}$.

Let now $C$ be an algebra and $\mathcal{T}$ a generalized standard family of stable tubes in $\Gamma_{C}$. Following [4], an algebra $B$ is said to be a quasi-tube enlargement of $C$ using modules from $\mathcal{T}$ if there is a finite sequence of algebras $A_{0}=C, A_{1}, \ldots, A_{m}=B$ such that, for each $0 \leq j<m, A_{j+1}$ is obtained from $A_{j}$ by an admissible operation of 
type $(\operatorname{ad} 1),(\operatorname{ad} 2),\left(\operatorname{ad} 1^{*}\right)$, or $\left(\operatorname{ad} 2^{*}\right)$, with pivot either in a stable tube of $\mathcal{T}$ or in a quasi-tube of $\Gamma_{A_{j}}$ obtained from a stable tube of $\mathcal{T}$ by means of the sequence of admissible operations (of types $\left.(\operatorname{ad} 1),(\operatorname{ad} 2),\left(\operatorname{ad} 1^{*}\right),\left(\operatorname{ad} 2^{*}\right)\right)$ done so far. We note that a tubular extension (respectively, tubular coextension) of $C$ (in the sense of [25]), using modules from $\mathcal{T}$, is just an enlargement of $C$ invoking only admissible operations of type $(\operatorname{ad} 1)\left(\right.$ respectively, of type $\left.\left(\operatorname{ad} 1^{*}\right)\right)$.

Proposition 3.3. Let $B$ be a quasi-tube enlargement of an algebra $C$ using modules from a generalized standard family $\mathcal{T}$ of stable tubes of $\Gamma_{C}$, and $\mathcal{C}$ the family of components of $\Gamma_{B}$ obtained from $\mathcal{T}$ by means of admissible operations leading from $C$ to $B$. Then $\mathcal{C}$ is a generalized standard family of quasi-tubes of $\Gamma_{B}$.

For the proof of the above proposition we refer to [3, Lemma 2.2, 2.3] and [22, Theorem $\mathrm{C}]$. We end this section with the following consequence for a canonical algebra $C$ [27, Theorem XV.3.9].

Proposition 3.4. Let $C$ be a canonical algebra and $\mathcal{T}^{C}$ the canonical $\mathbb{P}_{1}(K)$-family of standard stable tubes of $\Gamma_{C}$. For an algebra $A$ the following equivalences hold.

(i) $A$ is a $\mathcal{T}^{C}$-tubular extension of $C$ if and only if $A$ is a branch $\mathcal{T}^{C}$-extension of $C$.

(ii) $A$ is a $\mathcal{T}^{C}$-tubular coextension of $C$ if and only if $A$ is a branch $\mathcal{T}^{C}$-coextension of $C$.

Recall from [4, Section 2], 25, Section 4] that for a branch extension $B$ of a canonical algebra $C$ the Auslander-Reiten quiver $\Gamma_{B}$ has a disjoint union decomposition

$$
\Gamma_{B}=\mathcal{P}^{B} \vee \mathcal{T}^{B} \vee \mathcal{Q}^{B}
$$

where $\mathcal{P}^{B}=\mathcal{P}^{C}$ is a family of components consisting of $C$-modules and containing all indecomposable projective $C$-modules, $\mathcal{Q}^{B}$ is a family of components containing all indecomposable injective $B$-modules but no projective $B$-module, and $\mathcal{T}^{B}$ is a $\mathbb{P}_{1}(K)$-family $\left(\mathcal{T}_{\lambda}^{B}\right)_{\lambda \in \mathbb{P}_{1}(K)}$ of pairwise orthogonal standard ray tubes separating $\mathcal{P}^{B}$ from $\mathcal{Q}^{C}$. Respectively, for branch coextension $B, \mathcal{P}^{B}$ is a family of components containing all indecomposable projective $B$-modules but no injective $B$-modules, $\mathcal{Q}^{B}=\mathcal{Q}^{C}$ is a family of components consisting of $C$-modules and containing all indecomposable injective $C$-modules, and $\mathcal{T}^{B}$ is a $\mathbb{P}_{1}(K)$-family $\left(\mathcal{T}_{\lambda}^{B}\right)_{\lambda \in \mathbb{P}_{1}(K)}$ of pairwise orthogonal standard coray tubes separating $\mathcal{P}^{B}$ from $\mathcal{Q}^{C}$.

\section{Selfinjective orbit algebras}

In this section we recall needed background on selfinjective orbit algebras. 
Let $B$ be an algebra and $\mathcal{E}_{B}=\left\{e_{i} \mid 1 \leq i \leq n\right\}$ be a fixed set of orthogonal primitive idempotents of $B$ with $1_{B}=e_{1}+\ldots+e_{n}$. Then the repetitive category $\widehat{B}$ of $B$ is the category with $\widehat{\mathcal{E}}_{B}=\left\{e_{m, i} \mid m \in \mathbb{Z}, 1 \leq i \leq n\right\}$ as a set of objects of $\widehat{B}$ and the morphism spaces defined by

$$
\widehat{B}\left(e_{m, i}, e_{r, j}\right)=\left\{\begin{array}{cl}
e_{j} B e_{i}, & r=m \\
D\left(e_{i} B e_{j}\right), & r=m+1 \\
0, & \text { otherwise }
\end{array}\right.
$$

and the composition of morphisms given by the multiplication in $B$ and the canonical $B$-B-bimodule structure of $D(B)=\operatorname{Hom}_{K}(B, K)$. For each $m \in \mathbb{Z}$, we denote by $B_{m}$ the full subcategory of $\widehat{B}$ given by the objects $e_{m, i}$ for all $i \in\{1, \ldots, n\}$. Observe that $\widehat{B}$ is a selfinjective locally bounded $K$-category. An automorphism $\varphi$ of $\widehat{B}$ is said to be

- positive if, for each pair $(m, i) \in \mathbb{Z} \times\{1, \ldots, n\}$, we have $\varphi\left(e_{m, i}\right)=e_{p, j}$ for some $p \geq m$ and some $j \in\{1, \ldots, n\}$;

- rigid if, for each pair $(m, i) \in \mathbb{Z} \times\{1, \ldots, n\}$, we have $\varphi\left(e_{m, i}\right)=e_{m, j}$ for some $j \in\{1, \ldots, n\}$;

- strictly positive if it is positive but not rigid.

An important role is played by the Nakayama automorphism $\nu_{\widehat{B}}$ of $\widehat{B}$ which is defined by

$$
\nu_{\widehat{B}}\left(e_{m, i}\right)=e_{m+1, i}, \quad \text { for all }(m, i) \in \mathbb{Z} \times\{1, \ldots, n\} .
$$

Note that the Nakayama automorphism $\nu_{\widehat{B}}$ is a strictly positive automorphism of $\widehat{B}$. A group $G$ of automorphisms of $\widehat{B}$ is said to be admissible if it acts freely on the set $\widehat{\mathcal{E}}_{B}$ and has finitely many orbits.

Let $B$ be an algebra and $G$ be an admissible group of automorphisms of $\widehat{B}$. Following Gabriel [11, we may consider the finite orbit $K$-category $\widehat{B} / G$ defined as follows. The objects of $\widehat{B} / G$ are the elements $a=G x$ of the set $\widehat{\mathcal{E}}_{B} / G$ of $G$-orbits in $\widehat{\mathcal{E}}_{B}$ and the morphism spaces are given by

$$
(\widehat{B} / G)(a, b)=\left\{\left(f_{y, x}\right) \in \prod_{(x, y) \in a \times b} \widehat{B}(x, y) \mid g \cdot f_{y, x}=f_{g y, g x} \text { for all } g \in G, x \in a, y \in b\right\},
$$

for all objects $a, b$ of $\widehat{B} / G$. Then we have a canonical Galois covering functor $F: \widehat{B} \rightarrow \widehat{B} / G$ which assigns to each object $x$ of $\widehat{B}$ its $G$-orbit $G x$, and, for any objects $x$ of $\widehat{B}$ and $a$ of $\widehat{B} / G, F$ induces natural $K$-linear isomorphisms

$$
\bigoplus_{y \in \widehat{\mathcal{E}}_{B}, F y=a} \widehat{B}(x, y) \stackrel{\sim}{\longrightarrow}(\widehat{B} / G)(F x, a)
$$




$$
\bigoplus_{y \in \widehat{\mathcal{E}}_{B}, F y=a} \widehat{B}(y, x) \stackrel{\sim}{\longrightarrow}(\widehat{B} / G)(a, F x) .
$$

The finite dimensional algebra $\bigoplus_{a, b \in \widehat{\mathcal{E}} / G}(\widehat{B} / G)(a, b)$ associated to the orbit category $\widehat{B} / G$ is a selfinjective algebra, denoted by $\widehat{B} / G$ and called an orbit algebra of $\widehat{B}$, with respect to the admissible automorphism group $G$ of $\widehat{B}$. The group $G$ acts also on the category $\bmod \widehat{B}$ of right $\widehat{B}$-modules (identified with contravariant functors from $\widehat{B}$ to $\bmod K$ with finite support) by $g M=M \circ g^{-1}$ for any $M \in \bmod \widehat{B}$ and $g \in G$. Further, we have the push-down functor $F_{\lambda}: \bmod \widehat{B} \rightarrow \bmod \widehat{B} / G$ such that $F_{\lambda}(M)(a)=\bigoplus_{x \in a} M(x)$ for a module $M$ in $\bmod \widehat{B}$ and an object $a$ of $\widehat{B} / G$.

The following theorem is a consequence of [11, Lemma 3.5, Theorem 3.6].

ThEOREM 4.1. Let $B$ be an algebra and $G$ a torsion-free admissible group of $K$ linear automorphisms of $\widehat{B}$. Then

(i) The push-down functor $F_{\lambda}: \bmod \widehat{B} \rightarrow \bmod \widehat{B} / G$ induces an injection from the set of $G$-orbits of isomorphism classes of indecomposable modules in $\bmod \widehat{B}$ into the set of isomorphism classes of indecomposable modules in $\bmod \widehat{B} / G$.

(ii) The push-down functor $F_{\lambda}: \bmod \widehat{B} \rightarrow \bmod \widehat{B} / G$ preserves the AuslanderReiten sequences.

In general, the push-down functor $F_{\lambda}: \bmod \widehat{B} \rightarrow \bmod \widehat{B} / G$, associated to a Galois covering $F: \widehat{B} \rightarrow \widehat{B} / G$ is not dense (see [2]). Following [9], a repetitive category $\widehat{B}$ is said to be locally support-finite, if for any object $x$ of $\widehat{B}$, the full subcategory of $\widehat{B}$ given by the supports $\operatorname{supp} M$ of all indecomposable modules $M$ in $\bmod \widehat{B}$ with $M(x) \neq 0$, is finite. Here, by a support of a module $M$ in $\bmod \widehat{B}$ we mean the full subcategory of $\widehat{B}$ given by all objects $z$ with $M(z) \neq 0$.

The following theorem is a consequence of [10, Proposition 2.5] (see also [9, Theorem]).

THEOREM 4.2. Let $B$ be an algebra with locally support-finite repetitive category $\widehat{B}$, and $G$ be a torsion-free admissible group of automorphisms of $\widehat{B}$. Then the push-down functor $F_{\lambda}: \bmod \widehat{B} \rightarrow \bmod \widehat{B} / G$ is dense. In particular, $F_{\lambda}$ induces an isomorphism of the orbit translation quiver $\Gamma_{\widehat{B}} / G$ of the Auslander-Reiten quiver $\Gamma_{\widehat{B}}$ of $\widehat{B}$, with respect to the action of $G$, and the Auslander-Reiten quiver $\Gamma_{\widehat{B} / G}$ of $\widehat{B} / G$.

Let A be a selfinjective algebra, $I$ an ideal of $A, B=A / I$, and $e$ an idempotent of $A$ such that $e+I$ is the identity of $B$. We may assume that $e=e_{1}+\ldots+e_{n}$, where $\left\{e_{i} ; 1 \leq i \leq n\right\}$ is a complete set of orthogonal primitive idempotents of $A$ 
which are not contained in $I$. Then such an idempotent $e$ is uniquely determined by $I$ up to an inner automorphism of $A$, and we call it a residual identity of $B$ [31]. Note that $B \cong e A e / e I e$. For an ideal $I$ of a selfinjective algebra $A$, we consider its left annihilator $\ell_{A}(I)=\{a \in A \mid a x=0$ for any $x \in I\}$ and its right annihilator $r_{A}(I)=\{a \in A \mid x a=0$ for any $x \in I\}$. Following [31, (2.1)] the ideal $I$ is said to be deforming if $e I e=\ell_{e A e}(I)=r_{e A e}(I)$ and $A / I$ is triangular. The following lemma was proved in [34, Lemma 5.1].

LEMma 4.3. Let $A$ be a selfinjective algebra, $e$ an idempotent of $A$, and assume that $\ell_{A}(I)=I$ e or $r_{A}(I)=e I$. Then $e$ is a residual identity of the quotient algebra $A / I$.

Moreover, the following result was obtained in [31, Proposition 2.3].

Proposition 4.4. Let $A$ be a selfinjective algebra, $I$ an ideal of $A, B=A / I$, e a residual identity of $B$, and assume that $I e I=0$. Then the following conditions are equivalent.

(i) Ie is an injective cogenerator in $\bmod B$.

(ii) $e I$ is an injective cogenerator in $\bmod B^{o p}$.

(iii) $\ell_{A}(I)=I e$.

(iv) $r_{A}(I)=e I$.

Moreover, under these equivalent conditions, we have $\operatorname{soc} A \subseteq I$ and $e I e=\ell_{e A e}(I)=$ $r_{e A e}(I)$.

We end this section with the criterion which is fundamental in the proof of the main theorem (see [32, Section 3 and 4] and [34, Theorem 5.3]).

THEOREM 4.5. Let $A$ be a selfinjective algebra over an algebraically closed field $K$. The following conditions are equivalent.

(1) $A$ is isomorphic to an orbit algebra $\widehat{B} /\left(\varphi \nu_{\widehat{B}}\right)$, where $B$ is an algebra over $K$ with acyclic quiver $Q_{B}$ and $\varphi$ is a positive automorphism of $\widehat{B}$.

(2) There is an ideal $I$ of $A$ and an idempotent $e$ of $A$ such that

(i) $r_{A}(I)=e I$,

(ii) the quiver $Q_{A / I}$ of $A / I$ is acyclic.

Moreover, in this case, $B$ is isomorphic to $A / I$. 


\section{Selfinjective algebras of strictly canonical type}

The aim of this section is to introduce some results on selfinjective algebras of strictly canonical type. In particular, we give an answer to the question when the canonical family of quasi-tubes of such an algebra is generalized standard.

The following results were established in [17, Theorem 5.1].

THEOREM 5.1. Let $B$ be a branch extension (respectively, branch coextension) of a canonical algebra $C$. Then there exist algebras $C_{q}, B_{q}^{-}, B_{q}^{+}, B_{q}^{*}$ and $\bar{B}_{q}, q \in \mathbb{Z}$, and a decomposition

$$
\Gamma_{\widehat{B}}=\vee_{q \in \mathbb{Z}}\left(\mathcal{X}_{q} \vee \mathcal{C}_{q}\right)
$$

of the Auslander-Reiten quiver $\Gamma_{\widehat{B}}$ of $\widehat{B}$ such that the following statements hold:

(a) for each $q \in \mathbb{Z}, \mathcal{X}_{q}$ is a family of components of $\Gamma_{\widehat{B}}$ containing exactly one simple $\widehat{B}$-module $S_{q}$;

(b) for each $q \in \mathbb{Z}, \mathcal{C}_{q}$ is a family $\left(\mathcal{C}_{q}(\lambda)\right)_{\lambda \in \mathbb{P}_{1}(K)}$ of pairwise orthogonal standard quasi-tubes of $\Gamma_{\widehat{B}}$ with $s\left(\mathcal{C}_{q}(\lambda)\right)+p\left(\mathcal{C}_{q}(\lambda)\right)=r\left(\mathcal{C}_{q}(\lambda)\right)-1$, for any $\lambda \in \mathbb{P}_{1}(K)$;

(c) for each pair $p, q \in \mathbb{Z}$ with $p<q$, we have $H_{0 m}\left(\mathcal{X}_{q}, \mathcal{X}_{p} \vee \mathcal{C}_{p}\right)=0$ and $\operatorname{Hom}_{\widehat{B}}\left(\mathcal{C}_{q}, \mathcal{X}_{p} \vee \mathcal{C}_{p} \vee \mathcal{X}_{p+1}\right)=0$

(d) for each $q \in \mathbb{Z}, C_{q}$ is a canonical algebra, $B_{q}^{-}$is a maximal branch coextension of $C_{q}$ in $B_{q}^{*}, B_{q}^{+}$is a maximal branch extension of $C_{q}$ in $B_{q}^{*}$, and $B_{q}^{*}$ is a quasitube enlargement of $C_{q}$; moreover, $C_{q}, B_{q}^{-}, B_{q}^{+}, B_{q}^{*}$ and $\bar{B}_{q}$ are full convex subcategories of $\widehat{B}$ with $\widehat{B_{q}^{-}}=\widehat{B}=\widehat{B_{q}^{+}}, \nu_{\widehat{B}}\left(C_{q}\right)=C_{q+2}, \nu_{\widehat{B}}\left(B_{q}^{-}\right)=B_{q+2}^{-}$, $\nu_{\widehat{B}}\left(B_{q}^{+}\right)=B_{q+2}^{+}, \nu_{\widehat{B}}\left(B_{q}^{*}\right)=B_{q+2}^{*}, \nu_{\widehat{B}}\left(\bar{B}_{q}\right)=\bar{B}_{q+2}$.

(e) for each $q \in \mathbb{Z}, \mathcal{C}_{q}$ is the canonical $\mathbb{P}_{1}(K)$-family of quasi-tubes of $\Gamma_{B_{q}^{*}}$, obtained from the canonical $\mathbb{P}_{1}(K)$-family $\mathcal{T}_{q}^{-}$of coray tubes of $\Gamma_{B_{q}^{-}}$by infinite rectangle insertions, and from the canonical $\mathbb{P}_{1}(K)$-family $\mathcal{T}_{q}^{+}$of ray tubes of $\Gamma_{B_{q}^{+}}$by infinite rectangle coinsertions;

(f) for each $q \in \mathbb{Z}, \mathcal{X}_{q}$ consists of indecomposable $\bar{B}_{q}$-modules;

(g) for each $q \in \mathbb{Z}$, we have $\nu_{\widehat{B}}\left(\mathcal{X}_{q}\right)=\mathcal{X}_{q+2}$ and $\nu_{\widehat{B}}\left(\mathcal{C}_{q}\right)=\mathcal{C}_{q+2}$;

(h) for each $q \in \mathbb{Z}, \operatorname{Hom}_{\widehat{B}}\left(S_{q}, \mathcal{C}_{q}(\lambda)\right) \neq 0$ for all $\lambda \in \mathbb{P}_{1}(K)$, and Hom ${ }_{\widehat{B}}\left(S_{p}, \mathcal{C}_{q}\right)=0$ for $p \neq q$ in $\mathbb{Z}$;

(i) for each $q \in \mathbb{Z}, \operatorname{Hom}_{\widehat{B}}\left(\mathcal{C}_{q}(\lambda), S_{q+1}\right) \neq 0$ for all $\lambda \in \mathbb{P}_{1}(K)$, and $\operatorname{Hom}_{\widehat{B}}\left(\mathcal{C}_{q}, S_{p}\right)=$ 0 for $p \neq q+1$ in $\mathbb{Z}$;

(j) for each $q \in \mathbb{Z}$, we have $\Omega_{\widehat{B}}\left(\mathcal{C}_{q+1}^{s}\right)=\mathcal{C}_{q}^{s}$ and $\Omega_{\widehat{B}}\left(\mathcal{X}_{q+1}^{s}\right)=\mathcal{X}_{q}^{s}$. 
(k) $\widehat{B}$ is locally support-finite.

Moreover, there is the following description of torsion-free admissible groups of automorphisms of $\widehat{B}$ [17, Proposition 5.2].

Proposition 5.2. Let $B$ be a branch extension (respectively, branch coextension) of a canonical algebra $C$. Then there exists a strictly positive automorphism $\varrho_{\widehat{B}}$ of $\widehat{B}$ such that the following statements hold:

(i) $\varrho_{\widehat{B}}=\nu_{\widehat{B}}$ or $\varrho_{\widehat{B}}^{2}=\nu_{\widehat{B}}$;

(ii) every torsion-free admissible group $G$ of automorphisms of $\widehat{B}$ is an infinite cyclic group generated by a strictly positive automorphism $\sigma \varrho_{\widehat{B}}^{s}$, for some integer $s \geq 1$ and some rigid automorphism $\sigma$ of $\widehat{B}$.

Preserving the above notation, for a canonical algebra $C$, it follows from Proposition 3.3 that $\Gamma_{B}$ contains a generalized standard $\mathbb{P}_{1}(K)$-family $\mathcal{C}$ of quasi-tubes. We consider $\mathcal{C}=\mathcal{C}_{0}$ as a family of components of $\Gamma_{\widehat{B}}$.

Recall that, following [17], a selfinjective algebra $A$ of the form $\widehat{B} / G$, where $B$ is a branch extension (equivalently, branch coextension) of a canonical algebra $C$ and $G$ is an infinite cyclic group generated by a strictly positive automorphism of $\widehat{B}$, is called a selfinjective algebra of strictly canonical type. The structure and homological properties of the Auslander-Reiten quivers of selfinjective algebras of strictly canonical type were described in [17, Theorem 5.3]. In particular, for a selfinjective algebra $A$ of strictly canonical type its Auslander-Reiten quiver has a decomposition

$$
\Gamma_{A}=\bigvee_{q \in \mathbb{Z} / n \mathbb{Z}}\left(\mathcal{X}_{q}^{A} \vee \mathcal{C}_{q}^{A}\right)
$$

for some positive integer $n$, and, for each $q \in \mathbb{Z} / n \mathbb{Z}, \mathcal{C}_{q}^{A}=\left(\mathcal{C}_{q}^{A}(\lambda)\right)_{\lambda \in \mathbb{P}_{1}(K)}$ is a $\mathbb{P}_{1}(K)$-family of quasi-tubes with $s\left(\mathcal{C}_{q}^{A}(\lambda)\right)+p\left(\mathcal{C}_{q}^{A}(\lambda)\right)=r\left(\mathcal{C}_{q}^{A}(\lambda)\right)-1$ for each $\lambda \in \mathbb{P}_{1}(K)$, and $\mathcal{X}_{q}^{A}$ is a family of components containing exactly one simple module $S_{q}$. Moreover, we have the following proposition which is an immediate consequence of properties of push-down functor $F_{\lambda}: \bmod \widehat{B} \rightarrow \bmod A$.

Proposition 5.3. Let $A=\widehat{B} / G$ be a selfinjective algebra of strictly canonical type. Then $\mathcal{C}_{0}^{A}=F_{\lambda}\left(\mathcal{C}_{0}\right)$ is a $\mathbb{P}_{1}(K)$-family of quasi-tubes in $\Gamma_{A}$ maximally saturated by simple and projective modules.

We are now in a position to prove the following equivalence.

Proposition 5.4. Let $A=\widehat{B} / G$, where $B$ is a branch extension (respectively, coextension) of a canonical algebra $C$ with respect to the canonical $\mathbb{P}_{1}(K)$-family of stable tubes and $G$ an admissible group of automorphisms of $\widehat{B}$ generated by a strictly positive automorphism of $\widehat{B}$. Then the following statements are equivalent. 
(i) The canonical $\mathbb{P}_{1}(K)$-family $\mathcal{C}_{0}^{A}=F_{\lambda}\left(\mathcal{C}_{0}\right)$ of quasi-tubes of $\Gamma_{A}$ is generalized standard.

(ii) $G=\left(\varphi \nu_{\widehat{B}}\right)$, where $\varphi$ is strictly positive, or $G=\left(\varphi \nu_{\widehat{B}}\right)$, where $\varphi$ is rigid and $B$ is a canonical algebra.

Proof. Let $C$ be a canonical algebra and $\mathcal{T}^{C}$ the canonical $\mathbb{P}_{1}(K)$-family of pairwise orthogonal standard stable tubes of $\Gamma_{C}$. Since the classes of repetitive algebras of branch extensions and branch coextensions of $C$ with respect to the canonical $\mathbb{P}_{1}(K)$-family of stable tubes coincide (see [17, Section 4]), invoking Proposition 3.4, we may assume that $B$ is a branch coextension of $C$.

Let $A$ be an orbit algebra $\widehat{B} / G$, where $G$ is generated by a strictly positive automorphism $g$ of $\widehat{B}$. Following Theorem 5.1 (a) and (b), the Auslander-Reiten quiver $\Gamma_{\widehat{B}}$ of $\widehat{B}$ has a decomposition:

$$
\Gamma_{\widehat{B}}=\vee_{q \in \mathbb{Z}}\left(\mathcal{X}_{q} \vee \mathcal{C}_{q}\right),
$$

such that, for each $q \in \mathbb{Z}, \mathcal{X}_{q}$ is a family of components containing exactly one simple $\widehat{B}$-module $S_{q}$, and $\mathcal{C}_{q}$ is a family $\left(\mathcal{C}_{q}(\lambda)\right)_{\lambda \in \mathbb{P}_{1}(K)}$ of pairwise orthogonal standard quasitubes. We set $C=C_{0}$ and $B=B_{0}^{-}$. Applying now Theorem 5.1(e),(h) and (i), we know that there exist, for any $q \in \mathbb{Z}$, an indecomposable $C_{q}$-module in $\mathcal{C}_{q}$ with $S_{q+1}$ in its top and an indecomposable $C_{q+1}$-module in $\mathcal{C}_{q+1}$ which has $S_{q+1}$ in the socle. Hence $\operatorname{Hom}_{\widehat{B}}\left(\mathcal{C}_{0}, \mathcal{C}_{1}\right) \neq 0$.

Assume $\mathcal{C}_{0}^{A}=F_{\lambda}\left(\mathcal{C}_{0}\right)$ is a generalized standard family of quasi-tubes. Since $\nu_{\widehat{B}}\left(\mathcal{C}_{q}\right)=\mathcal{C}_{q+2}$ and $\operatorname{Hom}_{\widehat{B}}\left(\mathcal{C}_{0}, \mathcal{C}_{1}\right) \neq 0$, applying Proposition 5.2, we conclude that $g=\varphi \nu_{\widehat{B}}$, where $\varphi$ is a positive automorphism of $\widehat{B}$. Recall that $\mathcal{C}_{0}$ is the canonical $\mathbb{P}_{1}(K)$-family of quasi-tubes obtained from the canonical $\mathbb{P}_{1}(K)$-family $\mathcal{T}^{B}$ of coray tubes of $\Gamma_{B}$ by iterated infinite rectangle insertions (see Theorem $5.1(\mathrm{e})$ ). Observe that $\mathcal{C}_{0}$ contains no projective-injective modules if and only if $\mathcal{T}^{B}$ contains no injective modules, that is, $B=C$ (see [17, Section 2 and 3]). In this situation $\operatorname{Hom}_{\widehat{B}}\left(\mathcal{C}_{0}, \mathcal{C}_{2}\right)=0$, since $\operatorname{supp} \mathcal{C}_{0} \cap \operatorname{supp} \mathcal{C}_{2}=\operatorname{supp} \mathcal{C}_{0} \cap \operatorname{supp} \nu_{\widehat{B}}\left(\mathcal{C}_{0}\right)=\emptyset$. Suppose now that $\mathcal{C}_{0}$ contains a projective-injective module, equivalently $B \neq C$. Then by Theorem $5.1(\mathrm{~g}), \mathcal{C}_{p}$, for any even $p \in \mathbb{Z}$, contains a projective-injective module. Let $P$ be a projective-injective module which belongs to $\mathcal{C}_{2}$. Clearly, then $P / \operatorname{soc} P$ belongs to $\mathcal{C}_{2}$. From Theorem 5.1(j) we obtain that the simple socle soc $P$ of $P$ belongs to $\mathcal{C}_{1}$. Again, by Theorem $5.1(\mathrm{j})$, we get that the projective cover $P^{\prime}$ of soc $P$ belongs to $\mathcal{C}_{0}$, because $\operatorname{rad} P^{\prime}=\Omega_{\widehat{B}}(\operatorname{soc} P)$ belongs to $\mathcal{C}_{0}$. Hence, there is a non-zero homomorphism $f: P^{\prime} \rightarrow P$ which implies that $\operatorname{Hom}_{\widehat{B}}\left(\mathcal{C}_{0}, \mathcal{C}_{2}\right) \neq 0$ for $B \neq C$. Therefore, if $\varphi$ is rigid, then $B=C$. Summing up, we conclude that (i) implies (ii).

Assume now that (ii) holds. Suppose that $M, N$ are indecomposable $\widehat{B}$-modules belonging to $\mathcal{C}_{p}$, for some $p \in \mathbb{Z}$. From the description of subcategories $B_{q}^{-}, B_{q}^{+}$of $\widehat{B}$, 
we know that $\operatorname{supp} \mathcal{C}_{q} \cap \operatorname{supp} \mathcal{C}_{q+3}=\emptyset$ for any $q \in \mathbb{Z}$ (see the proof of [17, Theorem 5.1]). Then, by the assumption imposed on a strictly positive generator $g$ of the group $G$, we obtain that $\operatorname{supp}^{g^{i}} M \cap \operatorname{supp} N=\emptyset$ for any integer $i \neq 0$. Since the push-down functor $F_{\lambda}: \bmod \widehat{B} \rightarrow \bmod A$ is dense, there are the following natural isomorphisms of $K$-vector spaces

$$
\begin{aligned}
& \bigoplus_{i \in \mathbb{Z}} \operatorname{Hom}_{\widehat{B}}\left(g^{i} M, N\right) \cong \operatorname{Hom}_{A}\left(F_{\lambda}(M), F_{\lambda}(N)\right), \\
& \bigoplus_{i \in \mathbb{Z}} \operatorname{Hom}_{\widehat{B}}\left(M,{ }^{g^{i}} N\right) \cong \operatorname{Hom}_{A}\left(F_{\lambda}(M), F_{\lambda}(N)\right),
\end{aligned}
$$

for any indecomposable modules $M, N$ in $\bmod \widehat{B}$.

Let $X, Y$ be modules in $\mathcal{C}_{0}^{A}=F_{\lambda}\left(\mathcal{C}_{0}\right)$. Then $X=F_{\lambda}(M), Y=F_{\lambda}(N)$ for some $M \in \mathcal{C}_{p}, N \in \mathcal{C}_{q}$, and clearly $F_{\lambda}\left(\mathcal{C}_{p}\right)=F_{\lambda}\left(\mathcal{C}_{q}\right)=\mathcal{C}_{0}$. Without loss of generality we may assume that $p=q=0$. Thus $\operatorname{Hom}_{A}(X, Y) \cong \bigoplus_{i \in \mathbb{Z}} \operatorname{Hom}_{\widehat{B}}\left(M,{ }^{i} N\right) \cong$ $\operatorname{Hom}_{\widehat{B}}(M, N)$. Since $\mathcal{C}_{0}$ is a family of pairwise orthogonal standard quasi-tubes, we have $\operatorname{rad}_{\widehat{B}}^{\infty}(M, N)=0$, and hence $\operatorname{rad}_{A}^{\infty}(X, Y)=0$. This shows that (ii) implies (i).

\section{Proof of Theorem 1.1}

The implication (ii) $\Rightarrow$ (i) of the main theorem is an immediate consequence of Propositions 5.3 and 5.4 .

We prove now the implication (i) $\Rightarrow$ (ii). Suppose that $\mathcal{C}=\left(\mathcal{C}_{\lambda}\right)_{\lambda \in \Lambda}$ is a generalized standard family of quasi-tubes maximally saturated by simple and projective modules in the Auslander-Reiten quiver $\Gamma_{A}$ of a selfinjective algebra $A$. We recall that the annihilator of the family of components $\mathcal{C}=\left(\mathcal{C}_{\lambda}\right)_{\lambda \in \Lambda}$ is the intersection $\operatorname{ann}_{A}(\mathcal{C})=\bigcap_{X \in \mathcal{C}} \operatorname{ann}_{A}(X)$ of the annihilators of all indecomposable $A$-modules $X$ belonging to $\mathcal{C}$. Consider the quotient algebra $D=A / \operatorname{ann}_{A}(\mathcal{C})$. Then the family $\mathcal{C}$ is a generalized standard faithful family of quasi-tubes in $\Gamma_{D}$ maximally saturated by simple and projective modules. We claim that $D$ is a quasi-tube enlargement of a canonical algebra $C$. Namely, by definition, a quasi-tube is a connected translation quiver obtained from a stable tube by an iterated application of admissible operations ( $\operatorname{ad} 1),(\operatorname{ad} 2)$ and their dual versions. Assume that, for each $\lambda \in \Lambda$, a quasi-tube $\mathcal{C}_{\lambda}$ is obtained, as a translation quiver, from a stable tube $\mathcal{T}_{\lambda}$ by means of the above operations. This allows us to consider the family $T(\mathcal{C})=\bigcup_{\lambda \in \Lambda} T\left(\mathcal{C}_{\lambda}\right)$ of indecomposable modules in $\mathcal{C}$ such that, for each $\lambda \in \Lambda, T\left(\mathcal{C}_{\lambda}\right)$ corresponds to all vertices of the stable tube $\mathcal{T}_{\lambda}$. Let $C=D / \operatorname{ann}_{D}(T(\mathcal{C}))$ be a quotient algebra of $D$ by the annihilator $\operatorname{ann}_{D}(T(\mathcal{C}))$ of the family $T(\mathcal{C})$ given as the intersection $\bigcap_{Y \in T(\mathcal{C})} \operatorname{ann}_{D}(Y)$ of the annihilators of all modules belonging to $T(\mathcal{C})$. Then $D$ is 
a quasi-tube enlargement of $C$. Note that the modules from $T(\mathcal{C})$ form the family of stable tubes $\mathcal{T}^{C}=\left(\mathcal{T}_{\lambda}^{C}\right)_{\lambda \in \Lambda}$ in $\Gamma_{C}$, where $\mathcal{T}_{\lambda}^{C}=\mathcal{T}_{\lambda}$ for every $\lambda \in \Lambda$. Clearly, $\left(\mathcal{T}_{\lambda}^{C}\right)_{\lambda \in \Lambda}$ is a faithful generalized standard family of stable tubes in $\Gamma_{C}$ (maximally saturated by simple modules). Thus, invoking Theorem 2.2 , we conclude that $C$ is a canonical algebra and then $D$ is a quasi-tube enlargement of the canonical algebra $C$. In particular, $\Lambda=\mathbb{P}_{1}(K)$ and $\mathcal{T}^{C}$ is the separating canonical $\mathbb{P}_{1}(K)$-family $\left(\mathcal{T}_{\lambda}^{C}\right)_{\lambda \in \mathbb{P}_{1}(K)}=\left(\mathcal{T}_{\lambda}^{C}\right)_{\lambda \in \Lambda}$ of stable tubes in $\Gamma_{C}$. Hence, applying [4, (3.5)], we infer that there exists a unique maximal branch extension $B$ of $C$ inside $D$, which is obtained from $C$ by an iterated application of algebra operations of type $(\operatorname{ad} 1)$ (see also [22, Theorem $\mathrm{C}]$ ). Then the Auslander-Reiten quiver $\Gamma_{B}$ of $B$ contains a faithful $\mathbb{P}_{1}(K)$-family $\mathcal{T}^{B}=\left(\mathcal{T}_{\lambda}^{B}\right)_{\lambda \in \mathbb{P}_{1}(K)}$ of pairwise orthogonal generalized standard ray tubes (obtained from $\mathcal{T}^{C}=\left(\mathcal{T}_{\lambda}^{C}\right)_{\lambda \in \mathbb{P}_{1}(K)}$ by an iterated application of translation quiver operations of type $(\operatorname{ad} 1))$. Moreover, $D$ is obtained from $B$ by an iterated application of admissible algebra operations of types $\left(\operatorname{ad} 1^{*}\right),\left(\operatorname{ad} 2^{*}\right)$, and $\mathcal{C}$ from $\mathcal{T}^{B}$ by an iterated application of translation quiver operations of types $\left(\operatorname{ad} 1^{*}\right),\left(\operatorname{ad} 2^{*}\right)$. Then $B=D / \operatorname{ann}_{D}\left(\mathcal{T}^{B}\right)$, where $\mathcal{T}^{B}$ is taken as a family of modules. Thus we conclude that $B=A / \operatorname{ann}_{A}\left(\mathcal{T}^{B}\right)$ since $\operatorname{ann}_{D}\left(\mathcal{T}^{B}\right)=\operatorname{ann}_{A}\left(\mathcal{T}^{B}\right) \cap D$.

Let $I=\operatorname{ann}_{A}\left(\mathcal{T}^{B}\right)$. Then $B=A / I$. We will show now that $I$ satisfies the conditions (2) of Theorem 4.5. Observe that $Q_{A / I}=Q_{B}$ is acyclic, because $B$ is a $\mathcal{T}^{C}$-branch extension of the canonical algebra $C$.

By $J$ we shall denote the trace ideal of the family $\mathcal{T}^{B}$ in $A$, that is $J=\sum_{h} \operatorname{Im} h$, where $h \in \operatorname{Hom}_{A}\left(Y, A_{A}\right)$ for any $Y \in \mathcal{T}^{B}$. Since $A_{A}$ is of finite dimension over $K$, we obtain that $J$ is a finite sum $J=\sum_{i=1}^{s} \operatorname{Im} h_{i}$ for some homomorphisms $h_{i} \in \operatorname{Hom}_{A}\left(Y_{i}, A_{A}\right)$ with $Y_{i} \in \mathcal{T}^{B}$. Similarly, by $J^{\prime}$ we denote the trace ideal of the dual family $\mathrm{D}\left(\mathcal{T}^{B}\right)$ of left $A$-modules in $A$.

We may choose a complete set of pairwise orthogonal primitive idempotents $e_{1}, \ldots, e_{r}$ of $A$ such that $1_{A}=e_{1}+\ldots+e_{r}$ and $e=e_{1}+\ldots+e_{n}$, for some $n \leqslant r$, is a residual identity of $B=A / I$. Observe that $B \cong e A e / e I e$. We will show that $I$ is a deforming ideal of $A$ with $\ell_{A}(I)=I e$ and $r_{A}(I)=e I$. We will apply the strategy similar to the proof of [14, Theorem 7.14].

Proposition 6.1. We have $J \cup J^{\prime} \subseteq I$.

Proof. We know that $\mathcal{T}^{B}=\left(\mathcal{T}_{\lambda}^{B}\right)_{\lambda \in \mathbb{P}_{1}(K)}$ is a generalized standard family of ray tubes in $\Gamma_{B}$ and the generalized standard family $\mathcal{C}=\left(\mathcal{C}_{\lambda}\right)_{\lambda \in \mathbb{P}_{1}(K)}$ of quasi-tubes in $\Gamma_{A}$ is obtained from $\mathcal{T}^{B}$ by an iterated application of admissible translation quiver operations of types $\left(\operatorname{ad} 1^{*}\right)$ and $\left(\operatorname{ad} 2^{*}\right)$ corresponding to the admissible algebra operations of types $\left(\operatorname{ad} 1^{*}\right)$ and $\left(\operatorname{ad} 2^{*}\right)$ leading from $B$ to $D$. Then, applying arguments as in the proof of [14, Proposition 7.1], we prove the required inclusion $J \cup J^{\prime} \subseteq I$.

Applying arguments as in the proof of [14, Lemma 7.2] we obtain the following facts. 
Proposition 6.2. We have $\ell_{A}(I)=J, r_{A}(I)=J^{\prime}$ and $I=r_{A}(J)=\ell_{A}\left(J^{\prime}\right)$.

The following proposition is the key ingredient for proving that $I$ is a deforming ideal of $A$ such that $\ell_{A}(I)=I e$ and $r_{A}(I)=e I$.

Proposition 6.3. We have eIe $=e J e=e J^{\prime} e$. In particular, $(e I e)^{2}=0$.

Proof. Observe that $J$ is a right $B$-module since $1_{A}-e \in I$ implies that $J(1-e) \subseteq$ $J I=0$ and so $J=J e+J\left(1_{A}-e\right)=J e$. Hence $e J$ is an ideal of $e A e$ with $e J \subseteq e I e$, by Proposition 6.1 . We denote by $B^{\prime}$ the algebra $B^{\prime}=e A e / e J$. Note that $e$ is a residual identity of $B^{\prime}$.

Consider the canonical restriction functor $\operatorname{res}_{e}: \bmod A \rightarrow \bmod e A e$. Applying res $_{e}$ to the (generalized) standard family $\mathcal{C}=\left(\mathcal{C}_{\lambda}\right)_{\lambda \in \Lambda}$ of quasi-tubes maximally saturated by simple and projective modules in $\Gamma_{A}$ we obtain the family $\mathcal{T}^{B}$ of ray tubes in $\Gamma_{e A e}$. Moreover, $\mathcal{T}^{B}$ is sincere generalized standard in $\Gamma_{e A e}$. Further, $\mathcal{T}^{B}$ is also sincere generalized standard family for the quotient algebra $B^{\prime}=e A e / e J$ since $e J \subseteq e I e$. We shall show that, in fact, the algebras $B^{\prime}$ and $B$ are equal.

We shall compare the bound quivers $\left(Q_{B}, I_{B}\right),\left(Q_{B^{\prime}}, I_{B^{\prime}}\right)$ of algebras $B, B^{\prime}$, respectively. Since $Q_{B}$ is a subquiver of $Q_{B^{\prime}}$ with the same set of vertices, suppose there exists an arrow $\eta: x \rightarrow y$ in $Q_{B^{\prime}}$ which does not belong to $Q_{B}$. Recall that $Q_{B}$ is of the form

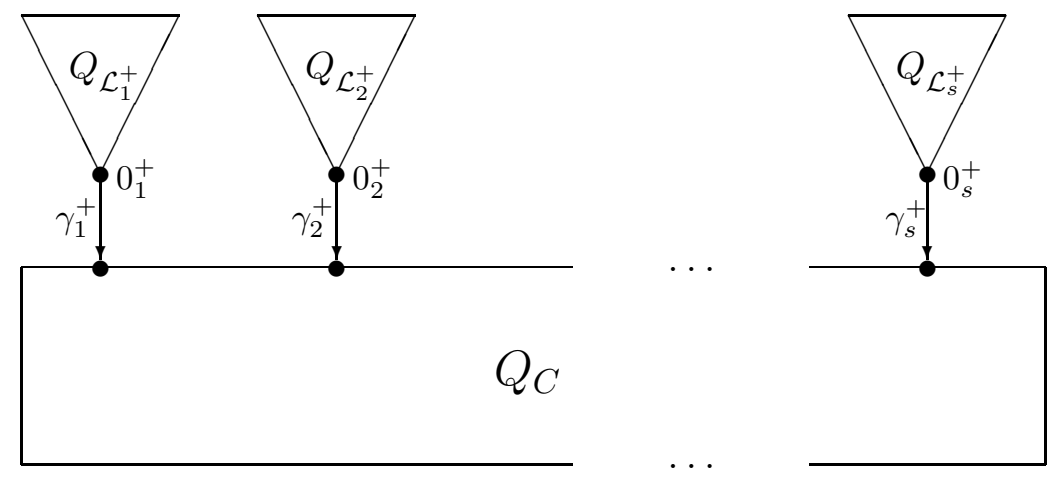

where $Q_{\mathcal{L}_{1}^{+}}, Q_{\mathcal{L}_{2}^{+}}, \ldots, Q_{\mathcal{L}_{s}^{+}}$are the quivers of the branches $\mathcal{L}_{1}^{+}, \mathcal{L}_{2}^{+}, \ldots, \mathcal{L}_{s}^{+}$, respectively, with the vertex $0_{i}^{+}$such that $\operatorname{rad} P\left(0_{i}^{+}\right)$, for $i \in\{1, \ldots, s\}$, are pairwise nonisomorphic modules lying on the mouths of stable tubes from canonical family $\mathcal{T}^{C}$ in $\Gamma_{C}$ (see [27, Chapter XV.3]). For $Q_{C}$ we shall use the notation from Section 2. By $B^{\prime \prime}$ we denote a quotient algebra of $B^{\prime}$ such that the set of arrows of $Q_{B^{\prime \prime}}$ consists of all arrows of $Q_{B}$ and additionally the arrow $\eta$. Hence we have a sequence of algebra epimorphisms $B^{\prime} \rightarrow B^{\prime \prime} \rightarrow B$. This implies that $\mathcal{T}^{B}$ is a sincere generalized standard family of ray tubes in $\Gamma_{B^{\prime \prime}}$. We have the following cases to consider.

(1) Assume that $x \in Q_{\mathcal{L}_{i}}$ for some $i \in\{1, \ldots, s\}$. Since $\mathcal{T}^{B}$ contains all projective $B$-modules $P(a)$ for $a \in Q_{\mathcal{L}_{i}}$ and $\mathcal{T}^{B}$ is a family of ray tubes in $\Gamma_{B^{\prime}}$, a projective 
$B$-module $P(x)$ is also a projective $B^{\prime}$-module. Therefore, if $\eta: x \rightarrow y$ in $Q_{B^{\prime}}$ then $\eta$ belongs to $Q_{B}$, a contradiction with an assumption imposed on $\eta$. If now $y \in Q_{\mathcal{L}_{i}}$, for some $i \in\{1, \ldots, s\}$, then there exists a homomorphism $f: P(y) \rightarrow P(x)$ in $\bmod B^{\prime}$ given by the formula $f(-)=\eta \cdot-$. Hence $\operatorname{Im} f=\eta e_{y} B^{\prime}=\eta e A e$, because $\mathcal{T}^{B}$ is a family of ray tubes in $\Gamma_{e A e}$. Therefore $\eta e A e \subseteq e J$, contradiction with the assumption that $\eta \in Q_{B^{\prime}}$.

(2) Let now $x \in Q_{C}$ and $y \in Q_{C} \backslash\{\omega\}$. Assume $y=(i, k)$ for some $i \in\{1, \ldots, m\}$ and $k \in\left\{1, \ldots, p_{i}-1\right\}$. Then $S(i, k)$ has in $\bmod B^{\prime \prime}$ a minimal injective presentation of the form

$$
0 \rightarrow S(i, k) \rightarrow I(i, k) \rightarrow I(i, k+1) \oplus I(x) \oplus I\left(0^{+}\right),
$$

where $I\left(0^{+}\right)=0$ if there is no branch extension of $C$ at $S(i, k)$. Then using the quasiinverse $\nu_{B^{\prime \prime}}^{-1}$ of the Nakayama functor $\nu_{B^{\prime \prime}}$, we obtain the following exact sequence

$$
0 \rightarrow P(i, k) \rightarrow P(i, k+1) \oplus P(x) \oplus P\left(0^{+}\right) \rightarrow \tau_{B^{\prime \prime}}^{-} S(i, k) \rightarrow 0,
$$

where we assume that $P\left(0^{+}\right)=0$ if $I\left(0^{+}\right)=0$. Then the socle of $\tau_{B^{\prime \prime}}^{-} S(i, k)$ contains an additional direct summand $S(x)$. Therefore, $\tau_{B^{\prime \prime}}^{-} S(i, k) \neq \tau_{B}^{-} S(i, k)$, and hence $\mathcal{T}_{B}$ is not a family of ray tubes in $\Gamma_{B^{\prime \prime}}$ and neither is in $\Gamma_{B^{\prime}}$, a contradiction. In the case of $y=0$, we repeat the above arguments for a nonsimple module $F_{i}$ from the mouth of a stable tube from $\mathcal{T}^{C}$.

(3) Assume that $x=(i, k)$, for some $i \in\{1, \ldots, m\}$ and $k \in\left\{1, \ldots, p_{i}-1\right\}$, and $y=\omega$. Then we show analogously that $\tau_{B^{\prime \prime}}^{-}\left(\tau_{B} S(x)\right) \neq S(x)$ since $B^{\prime \prime}$-module $\tau_{B^{\prime \prime}}^{-}\left(\tau_{B} S(x)\right)$ contains $\eta B$ as a submodule. Again, we get a contradiction.

(4) Let $\eta: \omega \rightarrow \omega$. By (1), (2) and (3) we conclude that $\eta, \alpha_{1, p_{1}}, \ldots, \alpha_{m, p_{m}}$ are all arrows that start at $\omega$ in $Q_{e A e}$. Denote by $\varrho_{i}$ the path $\alpha_{i, p_{i}} \ldots \alpha_{i, 2} \alpha_{i, 1}$, for any $i \in\{1, \ldots, m\}$. Observe that $\eta \varrho_{i}$ belongs to the $K$-vector space $e_{\omega} A e_{0}$ generated by $\varrho_{1}$ and $\varrho_{2}$, otherwise using the canonical restriction functor $\operatorname{res}_{e^{\prime}}: \bmod e A e \rightarrow \bmod e^{\prime} A e^{\prime}$ for $e^{\prime}=e_{\omega}+e_{0}$, we obtain that $e^{\prime} A e^{\prime}$ is a wild algebra (see arguments from the proof of Theorem 2.2 and [27, (XVIII.1.6)]). Assume $\eta \varrho_{i} \neq 0$ in $B^{\prime \prime}$, for some $i \in\{1, \ldots, m\}$. Then $\eta \varrho_{i}=a_{1} \varrho_{1}+a_{2} \varrho_{2}$ for some $a_{1}, a_{2} \in K$ such that $a_{1}^{2}+a_{2}^{2}>0$. Consider the nonsimple module $E^{(0)}$ from the mouth of the stable tube $\mathcal{T}_{0}^{C}$ from the family $\mathcal{T}^{C}$ (see Section 2). Note that $E_{\varrho_{2}}^{(0)}=0$ and hence $E_{a_{1} \varrho_{1}}^{(0)}=E_{\eta \varrho_{i}}^{(0)}=0$. But $E_{a_{1} \varrho_{1}}^{(0)}\left(e_{\omega}\right)=a_{1} \varrho_{1} \neq 0$ and we conclude that $\eta \varrho_{i}=0$ for any $i \in\{1, \ldots, m\}$. Let now $k_{i} \in\left\{0,1, \ldots, p_{i}\right\}$ be the minimal integer such that $\eta \alpha_{i, p_{i}} \ldots \alpha_{i, k_{i}} \neq 0$ in $B^{\prime \prime}$ where we put $\alpha_{i, p_{i}} \ldots \alpha_{i, 0}=e_{\omega}$. Then, for any $i \in\{1, \ldots, m\}$, there exists a nonzero homomorphism $f_{i}: S\left(i, k_{i}-1\right)\left[p_{i}-k_{i}+1\right] \rightarrow P(\omega)$ in $\bmod e A e$, where $S\left(i, k_{i}-1\right)\left[p_{i}-k_{i}+1\right]$ is a module of $\mathcal{T}^{C}$-length $p_{i}-k_{i}+1$ lying on a ray starting at mouth module $S\left(i, k_{i}-1\right)$ in $\mathcal{T}^{C}$. Note that $\operatorname{Im} f_{i}=\eta e A e$. Thus $\eta e A e \subset e J$ and $\eta \notin Q_{B^{\prime}}$.

(5) Let now $\eta_{1}, \eta_{2}, \ldots, \eta_{r}$, for some $r \geq 1$, be all arrows in $Q_{B^{\prime}}$ which start at 0 and end in $\omega$. 
Consider the Galois covering $F: \widetilde{B^{\prime}} \rightarrow B^{\prime}$ with an infinite cyclic group $\mathbb{Z}$. Then $\widetilde{B^{\prime}}$ is a locally bounded $K$-category and it follows from [7, Section 2] that $\widetilde{B^{\prime}} \cong K Q_{\widetilde{B^{\prime}}} / I_{\widetilde{B^{\prime}}}$, where $Q_{\widetilde{B^{\prime}}}$ is a connected, locally finite, acyclic quiver with $I_{\widetilde{B^{\prime}}}$ an admissible ideal of the path category $K Q_{\widetilde{B^{\prime}}}$ of $Q_{\widetilde{B^{\prime}}}$. Thus quiver $\widetilde{Q_{B^{\prime}}}$ is of the form

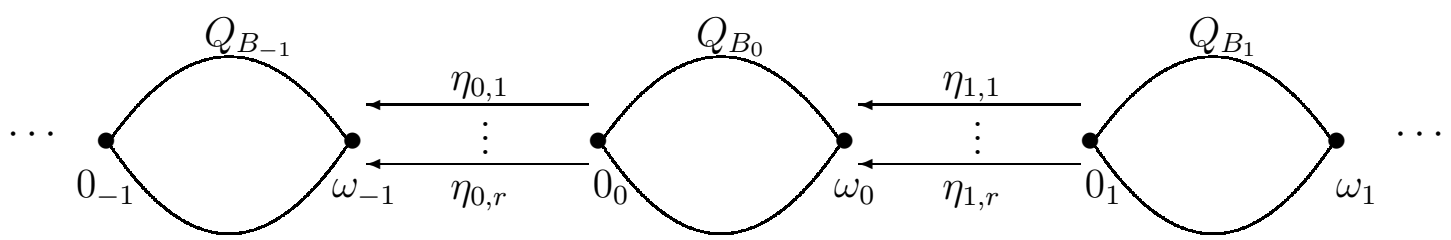

where, for all $k \in \mathbb{Z}$ and $1 \leq i \leq n$, we have $Q_{B_{k}}=Q_{B}, \eta_{k, i}: 0_{k} \rightarrow \omega_{k-1}$, and the generators of $I_{B_{k}}$ belong to the set generators of $I_{\widetilde{B^{\prime}}}$. By $B_{-}^{\prime}$ we denote the full subcategory of $\widetilde{B^{\prime}}$ whose objects are the objects of $B_{k}$ for all integer $k \leq 0$. Then the Auslander-Reiten quiver $B_{-}^{\prime}$ has a form

$$
\Gamma_{B_{-}^{\prime}}=\mathcal{P}^{B_{-}^{\prime}} \cup \mathcal{T}^{B_{0}} \cup \mathcal{Q}^{B_{0}}
$$

where $\mathcal{P}^{B_{-}^{\prime}}$ is a family of components containing all indecomposable modules $X$ such that $\operatorname{res}_{e}(X)$, for $e$ being the residual identity of $B_{0}$, is zero or belongs to the family $\mathcal{P}^{B_{0}}$ of $\Gamma_{B_{0}}, \mathcal{T}^{B_{0}}=\mathcal{T}^{B}$ and $\mathcal{Q}^{B_{0}}=\mathcal{Q}^{B}$. By $P$ we denote the projective $\widetilde{B^{\prime}}$-module $P_{\widetilde{B^{\prime}}}\left(0_{1}\right)$ at the vertex $0_{1}$ (vertex 0 in $\left.Q_{B_{1}}\right)$. Let now $R=B_{-}^{\prime}[\operatorname{rad} P]$ be a one-point extension of $B_{-}^{\prime}$ by the radical of $P$. Since $\mathcal{T}^{B_{0}}$ remains a family of ray tubes in $\Gamma_{R}$ (and in $\Gamma_{\widetilde{B^{\prime}}}$ ), we conclude that $\operatorname{rad} P \in \operatorname{add} \mathcal{Q}^{B_{0}}$ (see [27, Theorem XV.1.6]). Consider the projective cover $f: P^{\prime} \rightarrow \operatorname{rad} P$ of $\operatorname{rad} P$ in $\bmod B_{0}$. Then $f$ factorizes through the additive subcategory $\operatorname{add} \mathcal{T}^{B_{0}}$ of $\bmod B_{0}$, because $\mathcal{T}^{B_{0}}$ is a separating family of ray tubes in $\Gamma_{B_{0}}$. Therefore, there is a module $M \in \operatorname{add} \mathcal{T}^{B_{0}}$ and an epimorphism $h: M \rightarrow \operatorname{rad} P$ in $\bmod \widetilde{B^{\prime}}$. Further, there exists an epimorphism $h^{\prime}: M \rightarrow \operatorname{rad} P$ in $\bmod B^{\prime}$ because for the push-down functor $F_{\lambda}: \bmod \widetilde{B^{\prime}} \rightarrow \bmod B^{\prime}$ we have $F_{\lambda}(M)=M$ and $F_{\lambda}(\operatorname{rad} P)=\operatorname{rad} P$. Observe that by (1)-(4), $P$ is a projective $e A e$-module. Hence $\operatorname{Im} h^{\prime}=\sum_{i=1}^{r} \eta_{i} e A e$ and $\eta_{1}, \ldots, \eta_{r} \in e J$, a contradiction.

To sum up, we obtain that $e I e=e J$. Hence $\mathcal{T}^{B}$ is faithful generalized standard family of ray tubes in $\bmod B^{\prime}$ because $e I e / e J=\operatorname{ann}_{B^{\prime}}\left(\mathcal{T}^{B}\right)=0$. We show analogously that $e I e=J^{\prime} e$. Applying now Proposition 6.2, we have $(e I e)^{2}=e J e e I e=$ $e J e I e=(e J e) I e=e J I e=0$.

We note that although Proposition 6.3 is the analogue of Lemma 7.3 in [14] their proofs are different because the family $\mathcal{C}$ of quasi-tubes is assumed only to be generalized standard whereas in [14 the quasi-tubes in $\mathcal{C}$ consist of modules which do not lie on infinite short cycles. But having Proposition 6.3, we may proceed as 
in [14, Section 7], and, using Lemmas 7.4-7.12 of [14], prove the following analogue of [14, Proposition 7.13].

Proposition 6.4. We have $I e=J$, eI $=J^{\prime}$, and $e I e=J \cap J^{\prime}$.

This allows us to prove the desired proposition.

Proposition 6.5. $I$ is a deforming ideal of $A$ with $\ell_{A}(I)=I$ e and $r_{A}(I)=e I$.

Proof. From Proposition 6.2 and 6.4 we know that $\ell_{A}(I)=J=I e$ and $r_{A}(I)=$ $J^{\prime}=e I$. In particular, we have $I e I=0$. Therefore, applying Proposition 4.4, we get $e I e=\ell_{e A e}(I)=r_{e A e}(I)$. Since $Q_{A / I}=Q_{B}$ is acyclic, this shows that $I$ is a deforming ideal of $A$.

We complete now the proof of implication (i) $\Rightarrow$ (ii) of Theorem 1.1. Since the ideal $I$ and the idempotent $e$ satisfy condition (2) in Theorem 4.5, we conclude that $A$ is isomorphic to an orbit algebra $\widehat{B} /\left(\varphi \nu_{\widehat{B}}\right)$, where $\varphi$ is a positive automorphism of $\widehat{B}$. Finally, applying Proposition 5.4 we infer that either $\varphi$ is strictly positive or $\varphi$ is rigid and $B$ is a canonical algebra, as required in (ii).

\section{Examples}

The following examples illustrate the statements of Theorem 1.1 and Corollary 1.2.

EXAmPLE 7.1. Let $B=K Q_{B} / I_{B}$, where $Q_{B}$ is the quiver

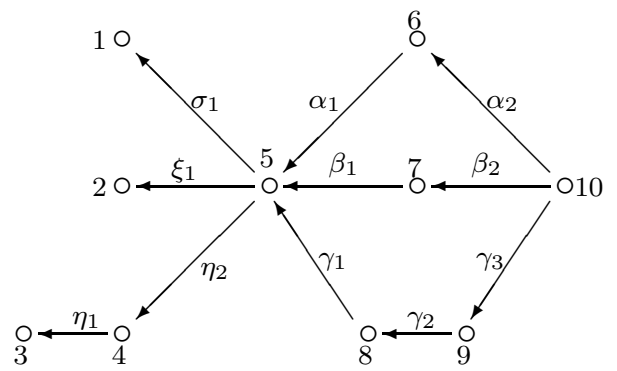

and $I_{B}$ is the ideal of the path algebra $K Q_{B}$ of $Q_{B}$ generated by the elements $\alpha_{1} \sigma_{1}, \beta_{1} \xi_{1}, \gamma_{1} \eta_{2}, \gamma_{3} \gamma_{2} \gamma_{1}+\alpha_{2} \alpha_{1}+\beta_{2} \beta_{1}$. Denote by $C$ the bound quiver algebra $C=K Q_{C} / I_{C}$, where $Q_{C}$ is the full subquiver of $Q$ given by the vertices $5,6,7,8$, 9,10 , and $I_{C}$ is the ideal in the path algebra $K Q_{C}$ of $Q_{C}$ generated by $\gamma_{3} \gamma_{2} \gamma_{1}+$ $\alpha_{2} \alpha_{1}+\beta_{2} \beta_{1}$. Then $C$ is the canonical algebra $C(\boldsymbol{p}, \boldsymbol{\lambda})$ with the weight sequence $\boldsymbol{p}=(2,2,3)$ and the parameter sequence $\boldsymbol{\lambda}=(\infty, 0,1)$. Further, $B$ is the branch coextension of $C$ in the sense of [27, XV.3]. Namely, $B=\left[E_{1}, \mathcal{L}_{1}, E_{2}, \mathcal{L}_{2}, E_{3}, \mathcal{L}_{3}\right] C$ with $E_{1}=E^{(\infty)} \in \mathcal{T}_{\infty}^{C}, E_{2}=E^{(0)} \in \mathcal{T}_{0}^{C}, E_{3}=E^{(1)} \in \mathcal{T}_{1}^{C}, \mathcal{L}_{1}$ the branch given by the vertex $1, \mathcal{L}_{2}$ the branch given by the vertex $2, \mathcal{L}_{3}$ the branch given by the 
vertices 3,4 and the arrow $\eta_{1}$. Consider the repetitive algebra $\widehat{B}$ of $B$. Then there exists a strictly positive automorphism $\varphi_{\widehat{B}}$ of $\widehat{B}$, with $\varphi_{\widehat{B}}^{2}=\nu_{\widehat{B}}$ such that, for any $k \in \mathbb{Z}, \varphi_{\hat{B}}\left(e_{k, l}\right)=e_{k, l+5}$, if $1 \leq l \leq 5$ and $\varphi_{\hat{B}}\left(e_{k, l}\right)=e_{k+1, l-5}=\nu_{\widehat{B}}\left(e_{k, l-5}\right)$, if $6 \leq l \leq 10$. Denote by $A$ the orbit algebra $\widehat{B} /\left(\varphi_{\widehat{B}}\right)$. Then $A$ is the bound quiver algebra $A=K Q / I$, where $Q$ is the quiver

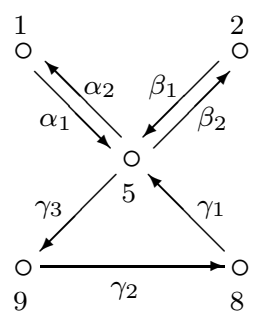

and $I$ is the ideal of $K Q$ generated by the elements $\gamma_{3} \gamma_{2} \gamma_{1}+\alpha_{2} \alpha_{1}+\beta_{2} \beta_{1}, \alpha_{1} \alpha_{2}, \beta_{1} \beta_{2}, \gamma_{1} \gamma_{3}$, $\alpha_{2} \alpha_{1} \beta_{2} \beta_{1}-\beta_{2} \beta_{1} \alpha_{2} \alpha_{1}$. Note that $A$ is a symmetric algebra but not a trivial extension of $C$. Hence from Corollary $1.2, \Gamma_{A}$ does not admit a generalized standard family of quasi-tubes maximally saturated by simple and projective modules. Indeed, by [17, Theorem 5.3] $\Gamma_{A}$ has a decomposition

$$
\Gamma_{A}=\mathcal{X}^{A} \vee \mathcal{C}^{A}
$$

where $\mathcal{C}^{A}=\left(\mathcal{C}^{A}(\lambda)\right)_{\lambda \in \mathbb{P}_{1}(K)}$ is the unique $\mathbb{P}_{1}(K)$-family of quasi-tubes of $\Gamma_{A}$ containing all simple modules and indecomposable projective modules, except the simple module $S(5)$ and the projective module $P(5)$ at the vertex 5 , which belong to $\mathcal{X}^{A}$. For $\lambda=1$ form the parameter sequence consider the module $E^{(1)}$ from the mouth of a stable tube $\mathcal{T}_{1}^{C}$ in $\Gamma_{C}$. Then $E^{(1)}$ belongs to $\mathcal{C}^{A}(1)$ and there is a nonzero homomorphism $f: E^{(1)} \rightarrow E^{(1)}$ which factors through $\operatorname{soc} E^{(1)} \cong S(5) \cong \operatorname{top} E^{(1)}$. Thus $f \in \operatorname{rad}^{\infty}\left(\mathcal{C}^{A}\right)$.

EXAmple 7.2. Consider the selfinjective algebra $A=\widehat{B} /\left(\varphi_{\widehat{B}}^{3}\right)$, where $B$ and $\varphi_{\widehat{B}}$ are as above. Then $A=K Q / I$ is the bound quiver algebra, where $Q$ is the quiver

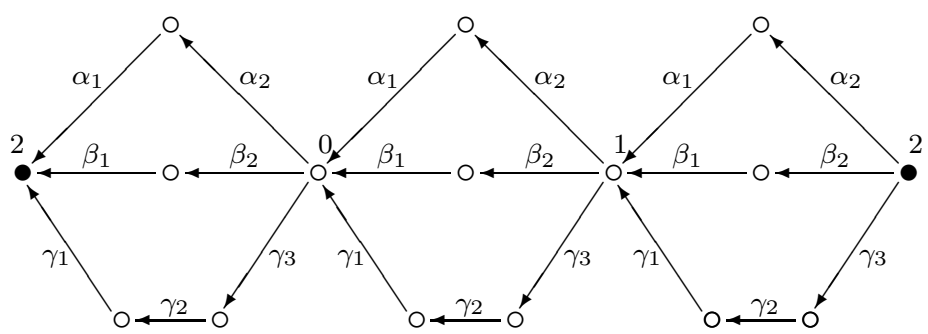

where we identify two vertices denoted by $\bullet$ and $I$ is the ideal of $K Q$ generated by the elements $\gamma_{3} \gamma_{2} \gamma_{1}+\alpha_{2} \alpha_{1}+\beta_{2} \beta_{1}, \alpha_{1} \alpha_{2}, \beta_{1} \beta_{2}, \gamma_{1} \gamma_{3}, \alpha_{2} \alpha_{1} \beta_{2} \beta_{1}-\beta_{2} \beta_{1} \alpha_{2} \alpha_{1}$. Again, 
by [17, Theorem 5.3] $\Gamma_{A}$ has a decomposition

$$
\Gamma_{A}=\mathcal{X}_{0}^{A} \vee \mathcal{C}_{0}^{A} \vee \mathcal{X}_{1}^{A} \vee \mathcal{C}_{1}^{A} \vee \mathcal{X}_{2}^{A} \vee \mathcal{C}_{2}^{A}
$$

where, for each $0 \leq i \leq 2, \mathcal{C}_{i}^{A}=\left(\mathcal{C}_{i}^{A}(\lambda)\right)_{\lambda \in \mathbb{P}_{1}(K)}$ is the $\mathbb{P}_{1}(K)$-family of quasi-tubes of $\Gamma_{A}, \mathcal{X}_{i}^{A}$ is a family of components containing exactly one simple module $S(i)$. Since $\varphi_{\widehat{B}}^{3}=\left(\varphi_{\widehat{B}}^{2}\right) \varphi_{\widehat{B}}=\nu_{\widehat{B}} \varphi_{\widehat{B}}$ and $\varphi_{\widehat{B}}$ is strictly positive automorphism of $\widehat{B}$, by Theorem 1.1 we get that $\mathcal{C}_{i}^{A}$, for some $0 \leq i \leq 2$, is generalized standard family of quasi-tubes maximally saturated by simple and projective modules. Further, applying Theorem 5.1 (see also [17, Theorem 5.1]), we have that $\mathcal{C}_{i}^{A}$, for each $0 \leq i \leq 2$, is a canonical family of quasi-tubes of $\Gamma_{B_{i}^{*}}$, where $B_{i}^{*}$ is a quasi-tube enlargement of canonical algebra $C_{i}=C$. Note that $B_{i}^{*}=K Q_{B_{i}^{*}} / I_{B_{i}^{*}}$, where $Q_{B_{i}^{*}}$ is of the form

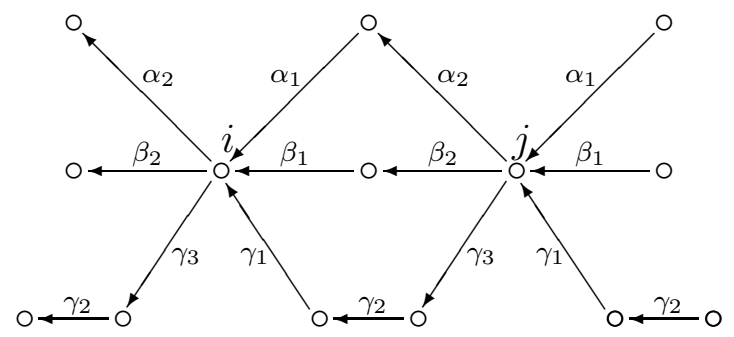

and $I_{B_{i}^{*}}$ is the ideal of $K Q_{B_{i}^{*}}$ generated by $\gamma_{3} \gamma_{2} \gamma_{1}+\alpha_{2} \alpha_{1}+\beta_{2} \beta_{1}, \alpha_{1} \alpha_{2}, \beta_{1} \beta_{2}, \gamma_{1} \gamma_{3}$ and $j \equiv i+1(\bmod 3)$. In conclusion, $\Gamma_{A}$ admits three generalized standard families $\mathcal{C}_{i}^{A}, 0 \leq i \leq 2$, of quasi-tubes maximally saturated by simple and projective modules.

ExAmple 7.3. Let $A=\widehat{B} /\left(\nu_{\widehat{B}}\right)$ be a selfinjective algebra, where $B=C$ is the canonical algebra as above. Then $A=K Q / I$ is the bound quiver algebra, where $Q$ is the quiver

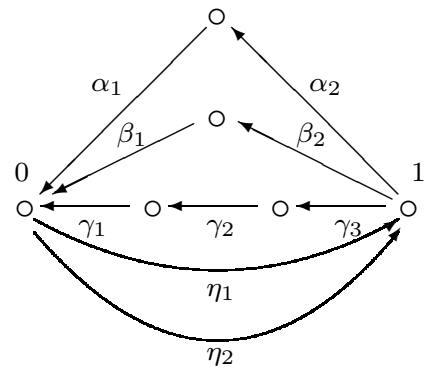

and $I$ is the ideal of $K Q$ generated by $\gamma_{3} \gamma_{2} \gamma_{1}+\alpha_{2} \alpha_{1}+\beta_{2} \beta_{1}, \eta_{1} \alpha_{2}, \eta_{2} \beta_{2}, \alpha_{1} \eta_{1}, \beta_{1} \eta_{2}, \eta_{1} \beta_{2} \beta_{1}-$ $\eta_{2} \alpha_{2} \alpha_{1}, \alpha_{2} \alpha_{1} \eta_{2}-\beta_{2} \beta_{1} \eta_{1}$. Following Theorem 5.1 and [17, Theorem 5.3], $\Gamma_{A}$ has a decomposition

$$
\Gamma_{A}=\mathcal{X}_{0}^{A} \vee \mathcal{C}_{0}^{A} \vee \mathcal{X}_{1}^{A} \vee \mathcal{C}_{1}^{A}
$$

where, for $i \in\{0,1\}, \mathcal{C}_{i}^{A}=\left(\mathcal{C}_{i}^{A}(\lambda)\right)_{\lambda \in \mathbb{P}_{1}(K)}$ is the $\mathbb{P}_{1}(K)$-family of quasi-tubes of $\Gamma_{A}$, $\mathcal{X}_{i}^{A}$ is a family of components containing exactly one simple module $S(i)$. Further, 
$\mathcal{C}_{0}^{A}$ is the canonical $\mathbb{P}_{1}(K)$-family of stable tubes of $\Gamma_{C}$ and $\mathcal{C}_{1}^{A}$ is the canonical $\mathbb{P}_{1}(K)$ family of quasi-tubes of $\Gamma_{B_{1}^{*}}$, where $B_{1}^{*}$ is the quasi-tube enlargement of Kronecker algebra $C_{1}$. Note that $\mathcal{C}_{1}^{A}$ contains projective $A$-module but no simple $A$-modules (see [17, Example 5.4]). Since $A=\widehat{B} /\left(\nu_{\widehat{B}}\right)$ is symmetric algebra, there is a nonzero homomorphism $f: P \rightarrow P$ for projective module $P \in \mathcal{C}_{1}^{A}$ which factorizes through simple module top $P$. Hence $f \in \operatorname{rad}_{A}^{\infty}$ and only $\mathcal{C}_{0}^{A}$ satisfies the condition of Theorem 1.1.

\section{REFERENCES}

[1] I. Assem, D. Simson and A. Skowroński, Elements of the Representation Theory of Associative Algebras 1: Techniques of Representation Theory, London Math. Soc. Stud. Texts 65 (Cambridge Univ. Press, 2006).

[2] I. Assem and A. Skowroński, On tame repetitive algebras, Fundamenta Math. 142 (1993), 59-84.

[3] I. Assem and A. Skowroński, Coils and multicoil algebras, in: Representation Theory of Algebras and Related Topics, Canad. Math. Soc. Conf. Proc. 19, Amer. Math. Soc. (1996), 1-24.

[4] I. Assem, A. Skowroński and B. Tomé, Coil enlargements of algebras, Tsukuba J. Math. 19 (1995), 453-479.

[5] M. Auslander, Representation theory of artin algebras II, Comm. Algebra 1 (1974), 269-310.

[6] J. Białkowski, A. Skowroński and K. Yamagata, Cartan matrices of symmetric algebras having generalized standard stable tubes, Osaka J. Math. 45 (2008), no. $1,15-171$.

[7] K. Bongartz and P. Gabriel, Covering spaces in representation theory, Invent. Math. 65 (1982), 331-378.

[8] F.U. Coelho, E.N. Marcos, H.A. Merklen and A. Skowroński, Module categories with infinite radical square zero are of finite type, Comm. Algebra 22 (1994), no. $11,4511-4517$.

[9] P. Dowbor and A. Skowroński, On Galois coverings of tame algebras, Arch. Math. (Basel) 44 (1985), 52-529. 
[10] P. Dowbor and A. Skowroński, Galois coverings of representation-infinite algebras, Comment. Math. Helv. 62 (1987), 311-337.

[11] P. Gabriel, The universal cover of a representation-finite algebra, in: Representation Theory I, Lecture Notes in Mathematics 903, Springer-Verlag, 1981, 68-105.

[12] D. Happel, Triangulated Categories in the Representation Theory of Finite Dimensional Algebras, London Math. Soc. Lecture Note Ser. 119, (Cambridge University Press, 1988).

[13] M. Karpicz, On selfinjective algebras without short cycles in the component quiver, Colloq. Math. 123 (2011), No.2, 219-232.

[14] M. Karpicz, A. Skowroński and K. Yamagata, On selfinjective artin algebras having generalized standard quasitubes, J. Pure Appl. Algebra 215 (2011), no. 11, 2738-2760.

[15] O. Kerner and A. Skowroński, On module categories with nilpotent infinite radical, Compositio Math. 77 (1991), no. , 313-333.

[16] O. Kerner, A. Skowroński and K. Yamagata, Invariance of selfinjective algebras of quasitilted type under stable equivalences, Manuscripta Math. 119 (2006), no. 3, 359-381.

[17] M. Kwiecień and A. Skowroński, Selfinjective algebras of strictly canonical type, Colloq. Math. 116 (2009), 47-103.

[18] M. Kwiecień and A. Skowroński, A classification of symmetric algebras of strictly canonical type, Colloq. Math. 116 (2009), no. 2, 249-271.

[19] M. Kwiecień and A. Skowroński, A characterization of selfinjective algebras of strictly canonical type, J. Algebra 323 (2010), no. 2, 473-508.

[20] S. Liu, The degrees of irreducible maps and the shapes of the Auslander-Reiten quivers, J. London Math. Soc. 45 (1992), 32-54.

[21] P. Malicki and A. Skowroński, Almost cyclic coherent components of an Auslander-Reiten quiver, J. Algebra 229 (2000), 695-749.

[22] P. Malicki and A. Skowroński, Algebras with separating almost cyclic coherent Auslander-Reiten components, J. Algebra 291 (2005), 208-237.

[23] P. Malicki and A. Skowroński, On the number of simple and projective modules in the quasi-tubes of self-injective algebras, Comm. Algebra 39(1), (2011), 322334. 
[24] Y. Ohnuki, K. Takeda and K. Yamagata, Automorphisms of repetitive algebras, J. Algebra 232 (2000), 708-724.

[25] C. M. Ringel, Tame Algebras and Integral Quadratic Forms, Lecture Notes in Math. 1099 (Springer Verlag, 1984).

[26] D. Simson and A. Skowroński, Elements of the Representation Theory of Associative Algebras 2: Tubes and Concealed Algebras of Euclidean Type, London Math. Soc. Stud. Texts 71 (Cambridge Univ. Press, 2007).

[27] D. Simson and A. Skowroński, Elements of the Representation Theory of Associative Algebras 3: Representation-Infinite Tilted Algebras, London Math. Soc. Stud. Texts 72 (Cambridge University Press, 2007).

[28] A. Skowroński, Generalized standard Auslander-Reiten components, J. Math. Soc. Japan 46 (1994), 517-543.

[29] A. Skowroński, Generalized canonical algebras and stable tubes, Colloq. Math. 90 (2001) 77-93.

[30] A. Skowroński, Selfinjective algebras: Finite and tame type, in: Trends in Representation Theory of Algebras and Related Topics, in: Contemp. Math., vol. 406, Amer. Math. Soc., 2006, pp. 169-238.

[31] A. Skowroński and K. Yamagata, Socle deformations of self-injective algebras, Proc. London Math. Soc. 72 (1996), 545-566.

[32] A. Skowroński and K. Yamagata, Galois coverings of selfinjective algebras by repetitive algebras, Trans. Amer. Math. Soc. 351 (1999), 715-734.

[33] A. Skowroński and K. Yamagata, On selfinjective artin algebras having nonperiodic generalized standard Auslander-Reiten components, Colloq. Math. 96 (2003), 233-244.

[34] A. Skowroński and K. Yamagata, Positive Galois coverings of selfinjective algebras, Adv. Math. 194 (2005), 398-436.

[35] A. Skowroński and K. Yamagata, Frobenius Algebras I. Basic Representation Theory, EMS Textbooks in Mathematics, European Mathematical Society Publishing House, Zürich, 2011.

[36] A. Skowroński and K. Yamagata, Frobenius Algebras II. Tilted and Hochschild Extension Algebras, EMS Textbooks in Mathematics, European Mathematical Society Publishing House, Zürich, 2017. 
[37] Y. Zhang, The structure of stable components, Canad. J. Math. 43 (1991), 652672. 